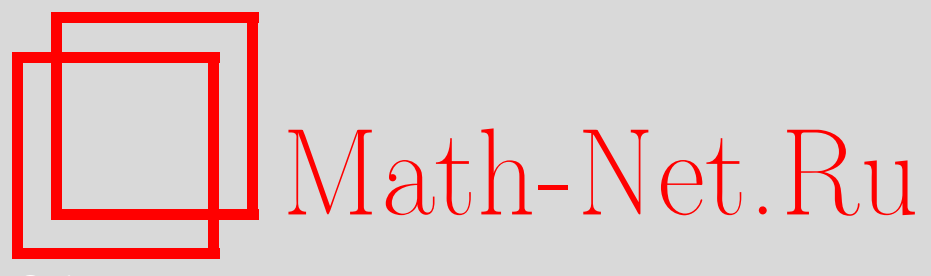

Д. Гиббон, Кватернионный репер, эволюционные уравнения Лагранжа и трехмерные уравнения Эйлера, $У M H$, 2007, том 62, выпуск 3, 47-72

DOI: https://doi.org/10.4213/rm6760

Использование Общероссийского математического портала Math-Net.Ru подразумевает, что вы прочитали и согласны с пользовательским соглашением http://www . mathnet.ru/rus/agreement

Параметры загрузки:

IP : 3.91 .87 .62

26 апреля 2023 г., 04:55:41

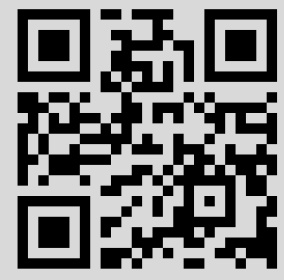


Посвящается памяти Виктора Юдовича (1934-2006), с которым автор обсуждал близкие идеи на ранней стадии работы

\title{
Кватернионный репер, эволюционные уравнения Лагранжа и трехмерные уравнения Эйлера
}

\begin{abstract}
Дж. Д. Гиббон
Кватернионы, которые были открыты Гамильтоном более 160 лет назад, в настоящее время широко используются в аэрокосмической промышленности, а также в компьютерной анимации при отслеживании ориентации, перемещения и вращения трехмерных объектов. В данном обзоре показано, что они приводят к весьма естественной ортонормированной системе координат, называемой кватернионной, которую можно использовать для описания динамики материальных точек лагранжевых течений, задаваемых соответствующими эволюционными уравнениями. Затем рассматривается приложение этого подхода к трехмерным уравнениям Эйлера для жидкости. Эта работа связана с проблемой о распространении особенностей решений уравнений Эйлера за конечное время. Делается обзор некоторых результатов на эту тему, включая теорему Била-Като-Майды и близкие к ней исследования о распространении вихрей, выполненные двумя командами авторов: Константином, Фефферманом и Майдой, а также Деном, Хоу и Ю. Показано, как кватернионный формализм обеспечивает альтернативные формулировки в терминах гессиана давления.

Библиография: 87 названий.
\end{abstract}

\section{СОДЕРЖАНИЕ}

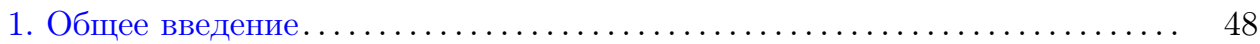

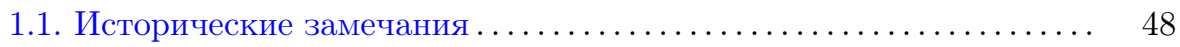

1.2. Приложение к гидродинамике ...................... 49

1.3. Взрыв решений в трехмерных уравнениях Эйлера ........... 51

1.4. Определение и свойства кватернионов ................ 53

2. Эволюционные уравнения Лагранжа и ортонормированный репер .... 54

3. Кватернионы и несжимаемые трехмерные уравнения Эйлера ........ 58

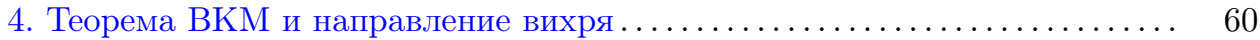

4.1. Работы Константина, Феффермана и Майды................ 61

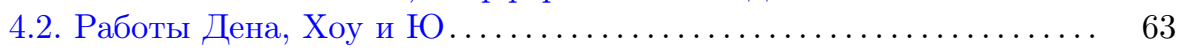

4.3. Непостоянство $\alpha_{p}$ и $\chi_{p}$ : кватернионы и направление вихря .... 64

5. Завершающий пример: уравнения несжимаемой идеальной МГД..... 66

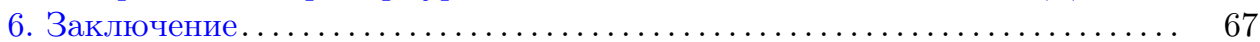

(C) Дж. Д. Гивьон, 2007 
Список литературы ..........................................

\section{1. Общее введение}

1.1. Исторические замечания. Все любят захватывающие истории: лихорадочное возбуждение Уильяма Роуана Гамильтона при открытии им 16 октября 1843 г. знаменитой формулы кватернионов, которую он использовал для ориентации своего телескопа; увековечение им этой формулы в виде надписи на мосту Broome (Brougham) в Дублине; а затем его долгая и в конечном счете бесплодная борьба за утверждение роли кватернионов в механике - все эти факты были забыты вплоть до недавних обращений к ним в [1], [2]. Имя Гамильтона весьма уважаемо в современной механике за смелость и глубину его идей, которые легли в основу симплектической геометрии [3]-[5]. Последующее признание его вклада и в классическую, и в квантовую математическую физику, а также в прикладную математику является неоспоримым. Однако в его собственное время работа Гамильтона по кватернионам вызвала бурную критику и даже откровенное высмеивание многими влиятельными учеными-современниками ${ }^{1}$. В конечном итоге кватернионы потерпели неудачу по сравнению с тензорными обозначениями Гиббса, которые в настоящее время являются общепринятыми для трехвекторного базиса.

По сути дела, правило умножения Гамильтона для кватернионов представляет собой суперпозицию вращений [6]-[11]. Это свойство было умело использовано в современных инерциальных системах управления в аэрокосмической промышленности для отслеживания ориентации и траекторий быстро перемещающихся и вращающихся спутников и космических кораблей. В книге [12] объясняется, как кватернионы работают на практике. Кватернионы также успешно используются в компьютерной графике при анимации сцен для контролирования ориентации объектов, вращающихся сложным образом. Во введении к своей прекрасной книге [2] Эндрю Хансон пишет следующие слова:

Несмотря на преимущество кватернионных форм для базисных уравнений контроля положения (ясно представленных в работах Кэли [6], Гамильтона [7], [8] и особенно Тейта [9]), что было замечено в аэронавтике и астронавтике, эта технология не проникала в область компьютерной анимации плоть до ключевой статьи [13], появившейся в трудах SIGGRAPH-1985. Важность этой работы состоит в том, что в ней была предложена концепция системы ориентации для движущихся трехмерных объектов и видеокамер, для чего требуется повышенная точность ориентации, отсутствие которой обнаруживается в стандартном методе углов Эйлера ${ }^{2}$, а в качестве решения аниматорам было предложено использовать кватернионы.

Критики Гамильтона в XIX веке были, конечно, правы в своих утверждениях, что для обращения с кватернионами необходима трехвекторная алгебра, однако использование их в аэро/астронавтике и в компьютерной анимации еще раз иллюстрирует многократно подтвержденную истину о том, что новый математический аппарат может не найти немедленного практического применения

\footnotetext{
Материалы этого обзора основаны на содержании лекции, прочитанной автором на конференции "Математическая гидродинамика", которая проходила в институте им. В. А. Стеклова в Москве в июне 2006 г.

${ }^{1}$ Примером может служить высказывание Кельвина, см. [1].

${ }^{2}$ Известный недостаток метода углов Эйлера заключается в том, что его точность падает в окрестности полюсов, в которых азимутальный угол не определен.
} 
и показаться кому-то слишком абстрактным или чрезмерно замысловатым, но в один прекрасный момент обнаруживается весьма полезное приложение именно для этого инструмента, которое и не снилось современникам его изобретателя.

1.2. Приложение к гидродинамике. Близкая связь кватернионов с вращениями твердого тела [9]-[11] указывает на возможность их использования в уравнениях Эйлера течения несжимаемой жидкости в качестве естественного языка, описывающего выравнивание вектора вихря по собственным векторам скорости деформации, которые отвечают за нелинейную эволюцию течения. Для трехмерного векторного поля $\boldsymbol{u}(\boldsymbol{x}, t)$ течения несжимаемой жидкости с давлением $p(\boldsymbol{x}, t)$ уравнения Эйлера имеют вид [14]-[18]

$$
\frac{D \boldsymbol{u}}{D t}=-\nabla p
$$

где материальная (субстанциональная) производная определяется по формуле

$$
\frac{D}{D t}=\frac{\partial}{\partial t}+\boldsymbol{u} \cdot \nabla
$$

На жидкость накладывается условие несжимаемости $\operatorname{div} \boldsymbol{u}=0$. Основная динамика заключается в эволюции матрицы градиента скорости $\nabla \boldsymbol{u}=\left\{u_{i, j}\right\}$, которая образуется при дифференцировании в (1.1):

$$
\frac{D u_{i, j}}{D t}=-u_{i, k} u_{k, j}-P_{i j},
$$

где $P_{i j}$ - элементы матрицы гессиана давления:

$$
P_{i j}=\frac{\partial^{2} p}{\partial x_{i} \partial x_{j}} .
$$

Условие несжимаемости $\operatorname{div} \boldsymbol{u}=0$ означает, что $\operatorname{Tr} u_{i, j}=0$. Последнее в примении к (1.3) дает соотношение

$$
\operatorname{Tr} P=\Delta p=-u_{i, k} u_{k, i}=\frac{1}{2} \omega^{2}-\operatorname{Tr} S^{2} .
$$

В формуле (1.5) $\boldsymbol{\omega}$ обозначает вихрь, а $S$ - матрицу деформации, элементы которой задаются выражениями

$$
S_{i j}=\frac{1}{2}\left(u_{i, j}+u_{j, i}\right) .
$$

Эта матрица является симметричной, и направление ее собственных векторов $\boldsymbol{e}_{i}$ играет фундаментальную роль в динамике уравнений Эйлера. Например, вихревые трубки и вихревые слои (вихри Бюргерса и др.) всегда имеют собственный вектор, выровненный по вектору вихря $\boldsymbol{\omega}$ [18].

В данном обзоре у нас нет возможности останавливаться на всех аспектах трехмерных уравнений Эйлера, в особенности рассматривать глубокие вопросы, связанные с понятиями слабых и обобщенных решений; читателю предлагается ознакомиться с книгой [15], где эти проблемы изучаются самым подробным образом. Здесь же все внимание будет сконцентрировано на той роли, которую играет естественный язык кватернионов для получения геометрической информации об эволюции функций $u_{i, j}$. Поскольку кватернионы особенно 
эффективны при вычислении ориентации вращающихся объектов, перемещающихся по трехмерным траекториям, они могут быть полезными для понимания того, как ведут себя общие течения Лагранжа, а также при нахождении ортогональных систем координат для описания траекторий материальных фрагментов таких течений. Эти фрагменты могут быть пассивными метками, перемещаемыми фоновым течением, или материальными точками (фрагментами) течения Лагранжа. Недавние эксперименты с турбулентными течениями позволяют теперь отслеживать траектории меток и материальных точек течений с большими числами Рейнольдса [19]-[28]: см. [19; рис. 1]. Для любой системы, в которой траектории представляются трехмерными пространственными кривыми, традиционный подход заключается в рассмотрении репера Френе траектории, который состоит из единичных векторов касательной, нормали и бинормали к траектории [2], [28]. На языке навигации это соответствует винтовому шагу, рысканию и крену движения. Однако при описании траектории с помощью репера Френе совершенно игнорируется динамика, которая порождает это движение. Делались попытки использования собственных векторов $\boldsymbol{e}_{i}$ матрицы $S$, но при этом возникли новые сложности, поскольку уравнения движения для $\boldsymbol{e}_{i}$ неизвестны [29]. В разделе 2 мы обсудим другой ортонормальный репер, связанный с движением каждой лагранжевой материальной точки, который называется кватернионным репером. Кватернионные реперы можно представлять себе движущимися вместе с материальными точками (элементами объема) Лагранжа, но их эволюция определяется уравнениями движения Эйлера. Преимущество такого подхода заключается в том, что лагранжеву динамику кватернионного репера можно связать с движением жидкости посредством гессиана давления $P$, который определен в (1.4).

Рассмотрим общую картину системы уравнений потока Лагранжа. Пусть $\boldsymbol{w}$ - контравариантный вектор, присоединенный к отмеченному фрагменту, который следует вдоль характеристических траекторий $d \boldsymbol{x} / d t=\boldsymbol{u}(\boldsymbol{x}, t)$ поля скорости $\boldsymbol{u}$. Рассмотрим абстрактное уравнение течения Лагранжа

$$
\frac{D \boldsymbol{w}}{D t}=\boldsymbol{a}(\boldsymbol{x}, t), \quad \frac{D}{D t}=\frac{\partial}{\partial 4 t}+\boldsymbol{u} \cdot \nabla,
$$

где материальная производная имеет стандартное определение; тогда $\boldsymbol{a}$ удовлетворяет уравнению Лагранжа

$$
\frac{D^{2} \boldsymbol{w}}{D t^{2}}=\frac{D \boldsymbol{a}}{D t}=\boldsymbol{b}(\boldsymbol{x}, t) .
$$

До этого момента мы имели дело лишь с кинематическими скоростями изменения при следовании вдоль характеристики скорости, порождающей траекторию $\boldsymbol{x}(t)$, которая задана уравнением $d \boldsymbol{x} / d t=\boldsymbol{u}(\boldsymbol{x}, t)$. Приведем примеры систем, которые можно представить в виде (1.7).

1. Если $\boldsymbol{w}$ представляет вихрь $\boldsymbol{\omega}=\operatorname{curl} \boldsymbol{u}$ уравнений Эйлера несжимаемой жидкости, то $\boldsymbol{a}=\boldsymbol{\omega} \cdot \nabla \boldsymbol{u}$ и $\operatorname{div} \boldsymbol{u}=0$. При повороте вектор $\boldsymbol{w}$ перейдет в $\boldsymbol{w} \equiv \widetilde{\boldsymbol{\omega}}=\rho_{0}^{-1}(\boldsymbol{\omega}+2 \boldsymbol{\Omega})$.

2. Для уравнений Эйлера баротропной сжимаемой жидкости (где давление $p=p(\rho)$ зависит только от плотности) $\boldsymbol{w} \equiv \boldsymbol{\omega}_{\boldsymbol{\rho}}=\rho^{-1} \boldsymbol{\omega}$. В этом случае $\boldsymbol{a}=\boldsymbol{\omega}_{\boldsymbol{\rho}} \cdot \nabla \boldsymbol{u}$ и $\operatorname{div} \boldsymbol{u}=0$.

3. Вектор $\boldsymbol{w}$ может представлять малый векторный линейный элемент $\boldsymbol{\delta} \boldsymbol{\ell}$, который смещается и растягивается фоновым потоком $\boldsymbol{u}$, и тогда $\boldsymbol{a}=$ 
$\boldsymbol{\delta} \boldsymbol{\ell} \cdot \nabla \boldsymbol{u}$. Например, в соответствиии с подмеченной $\Gamma$. К. Моффатом аналогией между магнитным полем $\boldsymbol{B}$ в теории идеальной несжимаемой МГД (магнитной гидродинамики) и вихрем [30], если $\boldsymbol{w}$ выбран так, что $\boldsymbol{w} \equiv \boldsymbol{B}$, то $\boldsymbol{a}=\boldsymbol{B} \cdot \nabla \boldsymbol{u}$, где $\operatorname{div} \boldsymbol{B}=0$. В более общей форме вектор $\boldsymbol{w}$ можно также представить переменными Элзассера $\boldsymbol{w}^{ \pm}=\boldsymbol{u} \pm \boldsymbol{B}$, и в этом случае $\boldsymbol{a}^{ \pm}=\boldsymbol{w}^{ \pm} \cdot \nabla \boldsymbol{u}$ с двумя материальными производными.

4. Семигеострофическую (SG) модель, используемую в физике атмосферы, можно также выразить в виде (1.7); например, можно выбрать $\boldsymbol{w}=\boldsymbol{x}$ и $\boldsymbol{a}=\boldsymbol{u}$, а $\boldsymbol{b}$ вычислить из SG-модели с помощью семигеострофического и агеострофического вкладов [31]-[33].

5. Для пассивной метки скорости $\boldsymbol{w}$, переносимой фоновым полем скоростей $\boldsymbol{u}$, ускорение метки будет $\boldsymbol{a}$ (см. [34], [16]).

В приведенных выше случаях 1-3 если $\boldsymbol{w}$ удовлетворяет стандартной эйлеровой форме

$$
\frac{D \boldsymbol{w}}{D t}=\boldsymbol{w} \cdot \nabla \boldsymbol{u}
$$

то для нахождения $\boldsymbol{b}$ по теореме Эртеля получаем, что (см. [35])

$$
\frac{D(\boldsymbol{w} \cdot \nabla \boldsymbol{\mu})}{D t}=\boldsymbol{w} \cdot \nabla\left(\frac{D \boldsymbol{\mu}}{D t}\right) .
$$

А это означает, что операторы $D / D t$ и $\boldsymbol{w} \cdot \nabla$ коммутируют между собой для любой дифференцируемой функции $\boldsymbol{\mu}(\boldsymbol{x}, t)$. Положив $\boldsymbol{\mu}=\boldsymbol{u}$, как это было сделано в [36], и обозначив ускорение потока через $\boldsymbol{Q}(\boldsymbol{x}, t)$, т.е. $D \boldsymbol{u} / D t=\boldsymbol{Q}(\boldsymbol{x}, t)$, получаем

$$
\frac{D^{2} \boldsymbol{w}}{D t^{2}}=\boldsymbol{w} \cdot \nabla\left(\frac{D \boldsymbol{u}}{D t}\right)=\boldsymbol{w} \cdot \nabla \boldsymbol{Q} .
$$

В каждом из рассмотренных выше случаев 1-3 функцию $\boldsymbol{Q}$ легко определить, и поэтому мы имеем функцию $\boldsymbol{b}$ :

$$
\frac{D \boldsymbol{a}}{D t}=\boldsymbol{w} \cdot \nabla \boldsymbol{Q}=: \boldsymbol{b}(\boldsymbol{x}, t)
$$

которая замыкает квартет векторов $(\boldsymbol{u}, \boldsymbol{w}, \boldsymbol{a}, \boldsymbol{b})$. В разделе 2 будет показано, что квартет векторов $(\boldsymbol{u}, \boldsymbol{w}, \boldsymbol{a}, \boldsymbol{b})$ полностью определяет естественный ортонормированный базис для лагранжевой динамики, который называется, как была сказано выше, кватернионным репером. По модулю вращения вокруг $\boldsymbol{w}$ кватернионный репер совпадает с репером Френе, присоединенным к характеристическим кривым $\boldsymbol{w}$. Равенство (1.10) получается отбрасыванием нелинейных членов порядка $O\left(|\boldsymbol{w}||\nabla \boldsymbol{u}|^{2}\right)$. Обычно этот результат приписывается Г. Эртелю [35], хотя он встречался в литературе и раньше, см. [36]-[40]. Теорема Эртеля позволяет найти $\boldsymbol{b}$ в случаях $1-3$, а в случае 4 вектор $\boldsymbol{b}$ определяется другим методом.

1.3. Взрыв решений в трехмерных уравнениях Эйлера. Общая картина лагранжевой эволюции и связанные с этим кватернионные реперы приведены в разделе 2. Далее в этой статье основное внимание сфокусировано на трехмерных несжимаемых уравнениях Эйлера (1.1) (см. раздел 3) и глобальном существовании решений (см. раздел 4).

Многие поколения математиков могли бы свидетельствовать об обманчивости видимой простоты уравнений Эйлера. Работа покойного В. Юдовича [41], 
который доказал существование и единственность слабых решений двумерных уравнений Эйлера при условии $\boldsymbol{\omega}_{0} \in L^{\infty}$ в неограниченной области, запомнится как важная веха в изучении эйлеровой динамики. В трехмерном случае пока известно лишь множество частных решений, которые можно записать с помощью простых функций [16]-[18], кроме того, нетривиальные результаты получены для слабых и обобщенных решений (см. [15]), тем не менее фундаментальный вопрос, становятся ли решения сингулярными за конечное время, остается без ответа. Говоря физическим языком, сингулярное поведение потенциально могло бы возникнуть, если бы вихрь вращался со все возрастающей скоростью и исчезал только при одновременном уменьшении его размеров до нуля за конечное время. Обзор некоторых аспектов проблемы сингулярности для трехмерной системы Эйлера содержится в последующих разделах данной статьи. Проблема регулярности для уравнений Навье-Стокса здесь рассматриваться не будет, а заинтересованный читатель может обратиться к работам [42]-[44].

Первый случай взрыва решений уравнений Эйлера был продемонстрирован в работах Дж. Т. Стюарта [45]-[47], где рассматривались решения трехмерного уравнения Эйлера, которые линейно зависели от двух переменных $x$ и $z$; для результирующих дифференциальных уравнений в остальных независимых переменных $y$ и $t$ сингулярность возникала за конечное время. Стюарт позднее показал, как метод характеристик позволяет построить полный класс сингулярных решений [45]. Этот тип сингулярности имеет бесконечную энергию, поскольку построенные решения являются линейными по двум направлениям $x$ и z. Похожим методом в работе [48] рассмотрен иной класс решений с бесконечной энергией, у которых третья компонента скорости линейна по $z$ и поэтому поле скорости принимает вид $\boldsymbol{u}=\left\{u_{1}(x, y, t), u_{2}(x, y, t), z \gamma(x, y, t)\right\}$. Это обобщает вихри Бюргерса [18] и представляет трубо- и кольцеподобные структуры, в зависимости от знака функции $\gamma(x, y, t)$. Веские численные данные о сингулярном поведении на периодическом пересечении, найденные в работе [49], были подтверждены аналитическим доказательством взрыва решения в работе [50]. Позднее в [51] с помощью метода, предложенного в [45], были найдены два явных сингулярных решения.

Теорема Била-Като-Майды (BKM) [52] была основным инструментом при изучении возможных сингулярностей эйлеровых решений с конечной энергией: одна из версий этой теоремы утверждает, что функция $\|\boldsymbol{\omega}\|_{\infty}$ должна удовлетворять неравенству для того, чтобы глобальное решение существовало вплоть до момента времени $T$,

$$
\int_{0}^{T}\|\boldsymbol{\omega}\|_{\infty} d \tau<\infty
$$

(более точную формулировку см. в разделе 4). Основное достоинство неравенства (1.13) заключается в том, что оно дает единый простой критерий, который легко проверять.

Помимо [53], где $\|\boldsymbol{\omega}\|_{\infty}$ заменено на $\|S\|_{\infty}$, и [54], где доказана ВМО-версия, имеется и несколько других уточнений теоремы BКМ. В частности, они принимают во внимание направления, в которых растут вихри. Работы [55] и [56], обзор которых дан в п. 4.1, заслуживают особого внимания. В них впервые была дана строгая математическая формулировка того, как несовпадение вихревых линий может приводить к возникновению сингулярности (или препятствовать этому явлению). Этот подход и его модификации легли в основу 
математических постановок задач для новых серий компьютерных экспериментов с уравнениями Эйлера. Пункт 4.2 посвящен обзору работ [57], [58], в которых установлены менее ограничительные критерии о линиях уровня. В п. 4.3 язык кватернионов рассматривается в качестве альтернативного метода исследования вихрей [59]. Этот путь приводит к другой теореме о направлении вихря, которая основана на матрице гессиана давления (1.4). Дальнейшее обсуждение этих вопросов и соответствующих литературных ссылок отложено до раздела 4.

1.4. Определение и свойства кватернионов. Для любого скаляра $p$ и произвольного трехмерного вектора $\boldsymbol{q}$ кватернион $\mathfrak{q}=[p, \boldsymbol{q}]$ определяется по формуле (для обозначения кватернионов будет использоваться готический шрифт)

$$
\mathfrak{q}=[p, \boldsymbol{q}]=p I-\sum_{i=1}^{3} q_{i} \sigma_{i},
$$

где $\left\{\sigma_{1}, \sigma_{2}, \sigma_{3}\right\}$ - три спиновые матрицы Паули, которые равны

$$
\sigma_{1}=\left(\begin{array}{cc}
0 & i \\
i & 0
\end{array}\right), \quad \sigma_{2}=\left(\begin{array}{cc}
0 & 1 \\
-1 & 0
\end{array}\right), \quad \sigma_{3}=\left(\begin{array}{cc}
i & 0 \\
0 & -i
\end{array}\right)
$$

а $I$ обозначает единичную матрицу размера $2 \times 2$. Матрицы $\left\{\sigma_{1}, \sigma_{2}, \sigma_{3}\right\}$ подчиняются соотношениям $\sigma_{i} \sigma_{j}=-\delta_{i j} I-\epsilon_{i j k} \sigma_{k}$. С помощью этих формул обосновывается следующее правило для некоммутативного умножения кватернионов:

$$
\mathfrak{q}_{1} \circledast \mathfrak{q}_{2}=\left[p_{1} p_{2}-\boldsymbol{q}_{1} \cdot \boldsymbol{q}_{2}, p_{1} \boldsymbol{q}_{2}+p_{2} \boldsymbol{q}_{1}+\boldsymbol{q}_{1} \times \boldsymbol{q}_{2}\right] .
$$

Легко устанавливается, что такое умножение кватернионов является ассоциативным. Главное свойство кватернионов, которое отсутствует у трехмерных векторов, состоит в том, что они имеют обратные элементы по умножению; обратный кватернион к $\mathfrak{q}$ равен $\mathfrak{q}^{*}=[p,-\boldsymbol{q}]$. Это означает, что $\mathfrak{q} \circledast \mathfrak{q}^{*}=\left[p^{2}+q^{2}, 0\right]=$ $\left(p^{2}+q^{2}\right)[1,0] ;$ в самом деле, $[1,0]$ есть скаляр, поэтому если $p^{2}+q^{2}=1$, то $\mathfrak{q}-$ это единичный кватернион $\hat{\mathfrak{q}}$.

Кватернион типа $\mathfrak{w}=[0, \boldsymbol{w}]$ называется чистым кватернионом, а произведение двух таких кватернионов можно записать в виде

$$
\mathfrak{w}_{1} \circledast \mathfrak{w}_{2}=\left[0, \boldsymbol{w}_{1}\right] \circledast\left[0, \boldsymbol{w}_{2}\right]=\left[-\boldsymbol{w}_{1} \cdot \boldsymbol{w}_{2}, \boldsymbol{w}_{1} \times \boldsymbol{w}_{2}\right] .
$$

Имеется кватернионная запись для оператора градиента $\nabla=[0, \nabla]$, которая применительно к чистому кватерниону $\mathfrak{u}=[0, \boldsymbol{u}]$ дает

$$
\boldsymbol{\nabla} \circledast \mathfrak{u}=[-\operatorname{div} \boldsymbol{u}, \operatorname{curl} \boldsymbol{u}] .
$$

Если поле $\boldsymbol{u}$ является бездивергентным, как в случае с несжимаемой жидкостью, то

$$
\boldsymbol{\nabla} \circledast \mathfrak{u}=[0, \boldsymbol{\omega}] .
$$

Такое чисто кватернионное представление вихря будет многократно использоваться в следующих разделах.

Как уже отмечалось в п. 1.1, кватернионы нашли применение в аэрокосмической промышленности и в компьютерной анимации, когда желательно избежать проблем с углами Эйлера. Сейчас мы кратко опишем кватернионные соотношения, а также приведем один из многочисленных способов, которые 
используются для описания вращения тел в богатой и вполне устоявшейся литературе по классической механике. Э. Т. Уиттекер [10] показывает, как соотносятся между собой кватернионы и параметры Кэли-Клейна [11], и дает точные формулы, связывающие эти параметры с углами Эйлера. Пусть $\hat{\mathfrak{q}}=[p, \boldsymbol{q}]-$ единичный кватернион с обратным $\hat{\mathfrak{q}}^{*}=[p,-\boldsymbol{q}]$, где $p^{2}+q^{2}=1$. Для чистого кватерниона $\mathfrak{r}=[0, \boldsymbol{r}]$ можно рассмотреть преобразование $\mathfrak{r} \rightarrow \mathfrak{r}^{\prime}=\left[0, \boldsymbol{r}^{\prime}\right]$ :

$$
\mathfrak{r}^{\prime}=\hat{\mathfrak{q}} \circledast \mathfrak{r} \circledast \hat{\mathfrak{q}}^{*} .
$$

Это ассоциативное произведение можно записать в явном виде

$$
\mathfrak{r}^{\prime}=\hat{\mathfrak{q}} \circledast \mathfrak{r} \circledast \hat{\mathfrak{q}}^{*}=\left[0,\left(p^{2}-q^{2}\right) \boldsymbol{r}+2 p(\boldsymbol{q} \times \boldsymbol{r})+2 \boldsymbol{q}(\boldsymbol{r} \cdot \boldsymbol{q})\right] .
$$

Выбрав $p= \pm \cos \frac{1}{2} \theta$ и $\boldsymbol{q}= \pm \hat{\boldsymbol{n}} \sin \frac{1}{2} \theta$, где $\hat{\boldsymbol{n}}$ - единичная нормаль к $\boldsymbol{r}$, находим, что

$$
\mathfrak{r}^{\prime}=\hat{\mathfrak{q}} \circledast \mathfrak{r} \circledast \hat{\mathfrak{q}}^{*}=[0, \boldsymbol{r} \cos \theta+(\hat{\boldsymbol{n}} \times \boldsymbol{r}) \sin \theta],
$$

где

$$
\hat{\mathfrak{q}}= \pm\left[\cos \frac{1}{2} \theta, \hat{\boldsymbol{n}} \sin \frac{1}{2} \theta\right] .
$$

Формула (1.22) описывает вращение на угол $\theta$ трехмерного вектора $\boldsymbol{r}$ вокруг его нормали $\hat{\boldsymbol{n}}$. Элементы единичного кватерниона $\hat{\mathfrak{q}}$ являются параметрами Кэли-Клейна, которые связаны с углами Эйлера. Все члены в (1.21) являются квадратичными по $p$ и $\boldsymbol{q}$ и, следовательно, как хорошо известно, обладают \pm -эквивалентностью.

Легко проверить, что знаменитые соотношения Гамильтона в терминах гиперкомплексных чисел $i^{2}=j^{2}=k^{2}=i j k=-1$ порождают правило умножения (1.16), если q записан в виде четырехмерного вектора $\mathfrak{q}=p+i q_{1}+j q_{2}+k q_{3}$. Статья [60] остается непревзойденным источником сведений о функциональных свойствах кватернионов; в ней, в частности, обсуждается, как для кватернионных функций выглядят аналоги различных результатов, хорошо известных для функций над полем комплексных чисел, таких, как уравнения Коши-Римана, теорема Коши и формула интегрирования вместе с разложением Лорана (но не конформные преобразования). Новые результаты по аналитическим свойствам таких функций можно найти в [61].

\section{2. Эволюционные уравнения Лагранжа и ортонормированный репер}

В этом разделе излагаются основы построения кватернионных реперов, которые приведены в работе [62]. Повторим еще раз эволюционные уравнения Лагранжа для векторного поля $\boldsymbol{w}$, которое удовлетворяет (1.7) и (1.8):

$$
\frac{D \boldsymbol{w}}{D t}=\boldsymbol{a}(\boldsymbol{x}, t), \quad \frac{D \boldsymbol{a}}{D t}=\boldsymbol{b}(\boldsymbol{x}, t) .
$$

Имея уравнения Лагранжа (2.1), определим скаляр $\alpha_{a}$ и трехмерный вектор $\chi_{a}$ по формулам ${ }^{3}$

$$
\alpha_{a}=|\boldsymbol{w}|^{-1}(\widehat{\boldsymbol{w}} \cdot \boldsymbol{a}), \quad \boldsymbol{\chi}_{a}=|\boldsymbol{w}|^{-1}(\widehat{\boldsymbol{w}} \times \boldsymbol{a}), \quad \boldsymbol{w} \neq 0 .
$$

\footnotetext{
${ }^{3}$ Роль нулевого вектора $\boldsymbol{w}=0$ остается невыясненной, хотя, как будет показано в разделе 3 , эта проблема полностью устраняется уравнениями течения Эйлера. Более подробно она обсуждается в [62].
} 


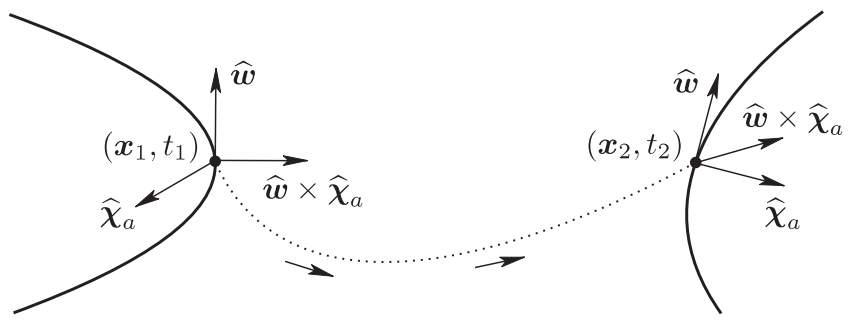

Рис. 1. Пунктирная линия обозначает траекторию метки $(\bullet)$ при ее движении из точки $\left(\boldsymbol{x}_{1}, t_{1}\right)$ в точку $\left(\boldsymbol{x}_{2}, t_{2}\right)$. Сплошные кривые обозначают характеристические кривые $\boldsymbol{w}=d \boldsymbol{x} / d s$, для которых $\widehat{\boldsymbol{w}}$ является единичным касательным вектором. Ориентация кватернионного репера $\left(\widehat{\boldsymbol{w}}, \widehat{\boldsymbol{\chi}}_{a}, \widehat{\boldsymbol{w}} \times \widehat{\boldsymbol{\chi}}_{a}\right)$ показана для двух пространственно-временных точек; заметим, что, в отличие от репера Френе, отвечающего траектории частицы, кватернионный репер отвечает характеристическим кривым $\boldsymbol{w}$.

Кроме того, определим величины $\alpha_{b}$ и $\boldsymbol{\chi}_{b}$ аналогично $\alpha_{a}$ и $\boldsymbol{\chi}_{a}$ в $(2.2)$, заменив $\boldsymbol{a}$ на $\boldsymbol{b}$. Вектор $\boldsymbol{a}$ можно представить в виде суммы двух векторов - параллельного и перпендикулярного $\boldsymbol{w}$ (то же делается для $\boldsymbol{b})$ :

$$
\boldsymbol{a}=\alpha_{a} \boldsymbol{w}+\boldsymbol{\chi}_{a} \times \boldsymbol{w}=\left[\alpha_{a}, \boldsymbol{\chi}_{a}\right] \circledast[0, \boldsymbol{w}] .
$$

Здесь кватернионное произведение используется самым естественным образом. По определению коэффициент роста $\alpha_{a}$ скалярной величины $|\boldsymbol{w}|$ подчиняется уравнению

$$
\frac{D|\boldsymbol{w}|}{D t}=\alpha_{a}|\boldsymbol{w}|
$$

а единичный вектор нормали $\widehat{\boldsymbol{w}}=\boldsymbol{w} w^{-1}$ удовлетворяет уравнению

$$
\frac{D \widehat{\boldsymbol{w}}}{D t}=\chi_{a} \times \widehat{\boldsymbol{w}}
$$

Теперь зададим кватернионы ${ }^{4}$

$$
\mathfrak{q}_{a}=\left[\alpha_{a}, \chi_{a}\right], \quad \mathfrak{q}_{b}=\left[\alpha_{b}, \chi_{b}\right],
$$

и пусть $\mathfrak{w}=[0, \boldsymbol{w}]$ обозначает чистый кватернион, удовлетворяющий эволюционному уравнению Лагранжа (2.1). Тогда исходное уравнение (2.1) можно переписать в эквивалентной кватернионной форме

$$
\frac{D \mathfrak{w}}{D t}=[0, \boldsymbol{a}]=\left[0, \alpha_{a} \boldsymbol{w}+\chi_{a} \times \boldsymbol{w}\right]=\mathfrak{q}_{a} \circledast \mathfrak{w} .
$$

Кроме того, если вектор $\boldsymbol{a}$ в уравнении (2.1) дифференцируем по Лагранжу, то разложение для $\boldsymbol{b}$, аналогичное разложению (2.3) для $\boldsymbol{a}$, дает равенство

$$
\frac{D^{2} \mathfrak{w}}{D t^{2}}=[0, \boldsymbol{b}]=\left[0, \alpha_{b} \boldsymbol{w}+\chi_{b} \times \boldsymbol{w}\right]=\mathfrak{q}_{b} \circledast \mathfrak{w} .
$$

\footnotetext{
${ }^{4}$ Опустив индексы $a, b$ и отнормировав результат, получаем, что параметры Кэли-Клейна примут вид $\hat{\mathfrak{q}}=[\alpha, \boldsymbol{\chi}]\left(\alpha^{2}+\chi^{2}\right)^{-1 / 2}$.
} 
Используя свойство ассоциативности и объединяя (2.8) и (2.7), получаем, что (при условии $|\boldsymbol{w}| \neq 0$ )

$$
\left(\frac{D \mathfrak{q}_{a}}{D t}+\mathfrak{q}_{a} \circledast \mathfrak{q}_{a}-\mathfrak{q}_{b}\right) \circledast \mathfrak{w}=0 .
$$

Тем самым установлено соотношение Риккати между $\mathfrak{q}_{a}$ и $\mathfrak{q}_{b}$ :

$$
\frac{D \mathfrak{q}_{a}}{D t}+\mathfrak{q}_{a} \circledast \mathfrak{q}_{a}=\mathfrak{q}_{b} .
$$

Это соотношение тесно связано с ортогональным кватернионным репером ${ }^{5}$ $\left(\widehat{\boldsymbol{w}}, \widehat{\boldsymbol{\chi}}_{a}, \widehat{\boldsymbol{w}} \times \widehat{\boldsymbol{\chi}}_{a}\right)$, уравнения движения которого задаются следующей теоремой.

ТЕОРема 1 [62]. Для системы уравнений (2.1), связывающих векторы $\boldsymbol{w}, \boldsymbol{a}$ u $\boldsymbol{b}$, ортонормированный кватернионный репер $\left(\widehat{\boldsymbol{w}}, \widehat{\boldsymbol{\chi}}_{a}, \widehat{\boldsymbol{w}} \times \widehat{\boldsymbol{\chi}}_{a}\right) \in S O(3)$ имеет лагранжеву производную по времени, которая выражается формулами

$$
\begin{aligned}
\frac{D \widehat{\boldsymbol{w}}}{D t} & =\mathcal{D}_{a b} \times \widehat{\boldsymbol{w}}, \\
\frac{D\left(\widehat{\boldsymbol{w}} \times \widehat{\boldsymbol{\chi}}_{a}\right)}{D t} & =\mathcal{D}_{a b} \times\left(\widehat{\boldsymbol{w}} \times \widehat{\boldsymbol{\chi}}_{a}\right), \\
\frac{D \widehat{\boldsymbol{\chi}}_{a}}{D t} & =\mathcal{D}_{a b} \times \widehat{\boldsymbol{\chi}}_{a},
\end{aligned}
$$

где угловой вектор скорости Дарбу $\mathcal{D}_{a b}$ определяется равенством

$$
\mathcal{D}_{a b}=\chi_{a}+\frac{c_{b}}{\chi_{a}} \widehat{\boldsymbol{w}}, \quad c_{b}=\widehat{\boldsymbol{w}} \cdot\left(\widehat{\boldsymbol{\chi}}_{a} \times \boldsymbol{\chi}_{b}\right),
$$

а $\boldsymbol{\chi}_{a}$ определено в (2.2), определение $\chi_{b}$ аналогично.

ЗАмечАниЕ. Ориентация репера контролируется вектором Дарбу $\mathcal{D}_{a b}$, который лежит на двумерной плоскости. В свою очередь, этот вектор управляется величиной $c_{b}=\widehat{\boldsymbol{w}} \cdot\left(\widehat{\boldsymbol{\chi}}_{a} \times \boldsymbol{\chi}_{b}\right)$, для которой вектор $\boldsymbol{b}$ должен быть известен. Ввиду этих обстоятельств появляется возможность численного решения уравнений (2.11)-(2.14) для конкретных траекторий.

ДокАЗАТЕЛьство. Чтобы найти выражение для лагранжевой производной по времени компонент репера $\left(\widehat{\boldsymbol{w}}, \widehat{\boldsymbol{\chi}}_{a}, \widehat{\boldsymbol{w}} \times \widehat{\boldsymbol{\chi}}_{a}\right)$, необходимо знать производную от $\widehat{\chi}_{a}$. Для нахождения этой величины можно использовать тот факт, что трехмерный вектор $\boldsymbol{b}$ допускает разложение в этом ортонормированном базисе в виде линейной комбинации

$$
w^{-1} \boldsymbol{b}=\alpha_{b} \widehat{\boldsymbol{w}}+c_{b} \widehat{\boldsymbol{\chi}}_{a}+d_{b}\left(\widehat{\boldsymbol{w}} \times \widehat{\chi}_{a}\right),
$$

где величина $c_{b}$ определена в $(2.14)$ и $d_{b}=-\left(\widehat{\chi}_{a} \cdot \chi_{b}\right)$. Трехмерный вектор $\boldsymbol{\chi}_{b}=w^{-1}(\widehat{\boldsymbol{w}} \times \boldsymbol{b})$ представляется в виде

$$
\chi_{b}=c_{b}\left(\widehat{\boldsymbol{w}} \times \widehat{\chi}_{a}\right)-d_{b} \widehat{\boldsymbol{\chi}}_{a}
$$

Для того чтобы найти производную по времени от $\widehat{\chi}_{a}$, воспользуемся трехмерной частью уравнения кватерниона $\mathfrak{q}_{a}=\left[\alpha_{a}, \boldsymbol{\chi}_{a}\right]$ в теореме 1 :

$$
\frac{D \chi_{a}}{D t}=-2 \alpha_{a} \chi_{a}+\chi_{b} \Rightarrow \frac{D \chi_{a}}{D t}=-2 \alpha_{a} \chi_{a}-d_{b},
$$

\footnotetext{
${ }^{5}$ В работе [2] показано, что кватернионный репер подобен реперу Бишопа в компьютерной графике.
} 
где $\chi_{a}=\left|\chi_{a}\right|$. Применяя (2.16) и (2.17), получаем

$$
\frac{D \widehat{\boldsymbol{\chi}}_{a}}{D t}=c_{b} \chi_{a}^{-1}\left(\widehat{\boldsymbol{w}} \times \widehat{\boldsymbol{\chi}}_{a}\right), \quad \frac{D\left(\widehat{\boldsymbol{w}} \times \widehat{\boldsymbol{\chi}}_{a}\right)}{D t}=\chi_{a} \widehat{\boldsymbol{w}}-c_{b} \chi_{a}^{-1} \widehat{\boldsymbol{\chi}}_{a},
$$

что влечет уравнения (2.11)-(2.14). Теорема 1 доказана.

Вопрос о том, как найти скорость изменения ускорения, представленного вектором $\boldsymbol{b}$, является особенно важным для вычисления траекторий пассивных меток, когда $\boldsymbol{b}$ не может быть вычислен по теореме Эртеля. Следующий результат описывает эволюцию $\mathfrak{q}_{b}$ в терминах трех произвольных скаляров.

ТеОРема 2 [62]. Производную Лагранжа по времени от $\mathfrak{q}_{b}$ можно выразить в виде

$$
\frac{D \mathfrak{q}_{b}}{D t}=\mathfrak{q}_{a} \circledast \mathfrak{q}_{b}+\lambda_{1} \mathfrak{q}_{b}+\lambda_{2} \mathfrak{q}_{a}+\lambda_{3} \mathbb{I}
$$

где $\lambda_{i}=\lambda_{i}(\boldsymbol{x}, t)$ - некоторые скалярные функиии $(\mathbb{I}=[1,0])$.

ДокАЗАТЕЛЬСтво. Чтобы получить (2.19), продифференцируем соотношение ортогональности $\boldsymbol{\chi}_{b} \cdot \widehat{\boldsymbol{w}}=0$ и используем лагранжеву производную от $\widehat{\boldsymbol{w}}$ :

$$
\frac{D \chi_{b}}{D t}=\chi_{a} \times \chi_{b}+s_{0}, \quad \text { где } \quad s_{0}=\mu \chi_{a}+\lambda \chi_{b} .
$$

Вектор $\boldsymbol{s}_{0}$ лежит в плоскости, перпендикулярной $\widehat{\boldsymbol{w}}$, в которой также лежат векторы $\chi_{a}$ и $\chi_{b}$, причем $\mu=\mu(\boldsymbol{x}, t)$ и $\lambda=\lambda(\boldsymbol{x}, t)-$ произвольные скаляры. Непосредственное дифференцирование равенства $\boldsymbol{\chi}_{b}=w^{-1}(\widehat{\boldsymbol{w}} \times \boldsymbol{b})$ дает соотношение

$$
w^{-1} \widehat{\boldsymbol{w}}\left(\boldsymbol{\chi}_{a} \cdot \boldsymbol{b}\right)+\boldsymbol{s}_{0}=-\alpha_{a} \boldsymbol{\chi}_{b}-\alpha_{b} \boldsymbol{\chi}_{a}+w^{-1} \widehat{\boldsymbol{w}}\left(\boldsymbol{\chi}_{a} \cdot \boldsymbol{b}\right)+w^{-1}\left(\widehat{\boldsymbol{w}} \times \frac{D \boldsymbol{b}}{D t}\right),
$$

которое можно переписать в виде

$$
\widehat{\boldsymbol{w}} \times\left\{\frac{D \boldsymbol{b}}{D t}-\alpha_{b} \boldsymbol{a}-\alpha_{a} \boldsymbol{b}\right\}=w \boldsymbol{s}_{0} .
$$

Это означает, что

$$
\frac{D \boldsymbol{b}}{D t}=\alpha_{b} \boldsymbol{a}+\alpha_{a} \boldsymbol{b}+\boldsymbol{s}_{0} \times \boldsymbol{w}+\varepsilon \boldsymbol{w},
$$

где $\varepsilon=\varepsilon(\boldsymbol{x}, t)$ - третий неизвестный скаляр в дополнение к $\mu$ и $\lambda$ в $(2.20)$. Значит, лагранжева производная от $\alpha_{b}=w^{-1}(\widehat{\boldsymbol{w}} \cdot \boldsymbol{b})$ равна

$$
\frac{D \alpha_{b}}{D t}=\alpha \alpha_{b}+\chi_{a} \cdot \chi_{b}+\varepsilon
$$

Следовательно, дифференциальные соотношения Лагранжа найдены теперь для $\chi_{b}$ и $\alpha_{b}$, однако платой за это является появление тройки неизвестных коэффициентов $\mu, \lambda$ и $\varepsilon$, которые можно переопределить следующим образом:

$$
\lambda=\alpha_{a}+\lambda_{1}, \quad \mu=\alpha_{b}+\lambda_{2}, \quad \varepsilon=-2 \chi_{a} \cdot \chi_{b}+\lambda_{2} \alpha_{a}+\lambda_{1} \alpha_{b}+\lambda_{3} .
$$

Подставляя новую тройку параметров в (2.19), видим, что уравнения (2.20) и (2.24) можно записать в кватернионной форме (2.19). Теорема 2 доказана. 
ТАБлицА 1. Представлены три способа нахождения поля $\boldsymbol{b}$ по тройке $(\boldsymbol{u}, \boldsymbol{w}, \boldsymbol{a})$. В третьей строке приведен результат (3.3), тогда как для второй строки $\boldsymbol{b}$ неизвестно.

\begin{tabular}{c|ccc|c}
$\boldsymbol{u}$ & $\boldsymbol{w}$ & $\boldsymbol{a}$ & $\boldsymbol{b}$ & Материальная производная \\
\hline Эйлер & $\boldsymbol{x}$ & $\boldsymbol{u}$ & $-\nabla p$ & $(1.2)$ \\
Эйлер & $\boldsymbol{u}$ & $-\nabla p$ & $?$ & $(1.2)$ \\
Эйлер & $\boldsymbol{w}$ & $S \boldsymbol{w}$ & $-P \boldsymbol{w}$ & $(1.2)$
\end{tabular}

\section{3. Кватернионы и несжимаемые трехмерные уравнения Эйлера}

Результаты предыдущего раздела о течениях Лагранжа непосредственно применяются к уравнениям Эйлера несжимаемой жидкости, однако для представления их в таком виде придется воспользоваться обратным хронологическим порядком, в котором они были впервые получены. Забегая вперед, отметим, что переменные $\alpha$ и $\chi$ в (3.4) для уравнений Эйлера и два связанных с ними дифференциальных уравнения (3.10) были выписаны впервые почти десять лет назад в [63], [64] без использования кватернионов. Позднее в [65] было обнаружено, что эти уравнения можно объединить в форме единого кватернионного уравнения Риккати. Наконец, в совсем еще свежей статье [59], а также в [62] все эти результаты были представлены в виде, принятом в настоящем обзоре. Поскольку решения трехмерного уравнения Эйлера быстро теряют гладкость, следует иметь в виду, что все наши дальнейшие рассуждения носят достаточно формальный характер.

В разделе 2 было показано, что для применения теоремы 1 необходима информация о квартете векторов $(\boldsymbol{u}, \boldsymbol{w}, \boldsymbol{a}, \boldsymbol{b})$. Обозначая $\boldsymbol{w} \equiv \boldsymbol{\omega}$, где $\boldsymbol{\omega}=\operatorname{curl} \boldsymbol{u}$, получим, что вектор растяжения вихря равен $\boldsymbol{a}=\boldsymbol{\omega} \cdot \nabla \boldsymbol{u}$. Значит, в этом случае векторные поля $\boldsymbol{w}$ и $\boldsymbol{u}$ не являются независимыми. Скалярное произведение $\boldsymbol{a}=\boldsymbol{\omega} \cdot \nabla \boldsymbol{u}$ выявляет только симметрическую часть матрицы вектора градиента $\nabla \boldsymbol{u}$, которая является матрицей деформации $S_{i j}=\frac{1}{2}\left(u_{i, j}+u_{j, i}\right)$, заданной в (1.6). С учетом равенства $\boldsymbol{a}=\boldsymbol{\omega} \cdot \nabla \boldsymbol{u}=S \boldsymbol{\omega}$ соответствующая тройка векторов имеет вид

$$
(\boldsymbol{u}, \boldsymbol{w}, \boldsymbol{a}) \equiv(\boldsymbol{u}, \boldsymbol{\omega}, S \boldsymbol{\omega})
$$

Чтобы найти поле $\boldsymbol{b}$, следует воспользоваться теоремой Эртеля из п. 1.2. Производная $D \boldsymbol{u} / D t$ в правой части равенства (1.1) (при $\boldsymbol{w}=\boldsymbol{\omega}$ ) удовлетворяет уравнению Эйлера $D \boldsymbol{u} / D t=-\nabla p$, т.е. получаем

$$
\boldsymbol{b}=\boldsymbol{\omega} \cdot \nabla\left(\frac{D \boldsymbol{u}}{D t}\right)=-P \boldsymbol{\omega}
$$

где $P$ - гессиан давления, определенный в (1.4). Квартет векторов, необходимый для применения теоремы 1 , имеет вид

$$
(\boldsymbol{u}, \boldsymbol{w}, \boldsymbol{a}, \boldsymbol{b}) \equiv(\boldsymbol{u}, \boldsymbol{\omega}, S \boldsymbol{\omega},-P \boldsymbol{\omega}) .
$$

В табл. 1 представлены три квартета $(\boldsymbol{u}, \boldsymbol{w}, \boldsymbol{a}, \boldsymbol{b})$ для уравнений Эйлера.

С учетом определений из раздела 2 скаляр $\alpha$ и трехмерный вектор $\chi$ задаются формулами

$$
\alpha=\widehat{\boldsymbol{\omega}} \cdot S \widehat{\boldsymbol{\omega}}, \quad \chi=\widehat{\boldsymbol{\omega}} \times S \widehat{\boldsymbol{\omega}}
$$


и по определению

$$
\alpha_{p}=\widehat{\boldsymbol{\omega}} \cdot P \widehat{\boldsymbol{\omega}}, \quad \chi_{p}=\widehat{\boldsymbol{\omega}} \times P \widehat{\boldsymbol{\omega}} .
$$

Величина $\alpha$ в (3.4) совпадает с ее выражением в [55], где она была определена формулой Био-Савара ${ }^{6}$. Вектор $\boldsymbol{a}=S \boldsymbol{\omega}$ можно разложить в сумму двух слагаемых - параллельного и перпендикулярного к $\boldsymbol{\omega}$ :

$$
S \boldsymbol{\omega}=\alpha \boldsymbol{\omega}+\chi \times \boldsymbol{\omega}=[\alpha, \chi] \circledast[0, \boldsymbol{\omega}] .
$$

По определению коэффициент роста $\alpha$ скалярной величины $|\boldsymbol{\omega}|$ и единичный вектор нормали $\widehat{\boldsymbol{\omega}}$ удовлетворяют уравнениям

$$
\frac{D|\boldsymbol{\omega}|}{D t}=\alpha|\boldsymbol{\omega}|, \quad \frac{D \widehat{\boldsymbol{\omega}}}{D t}=\boldsymbol{\chi} \times \widehat{\boldsymbol{\omega}},
$$

которые показывают, что коэффициент $\alpha$ управляет ростом или разрушением вихря, а $\boldsymbol{\chi}$ определяет частоту колебаний $\widehat{\boldsymbol{\omega}}$ около $S \boldsymbol{\omega}$. Теперь зададим кватернионы

$$
\mathfrak{q}=[\alpha, \chi], \quad \mathfrak{q}_{p}=\left[\alpha_{p}, \chi_{p}\right] .
$$

Эквивалентом уравнения Риккати (2.10) является уравнение ${ }^{7}$

$$
\frac{D \mathfrak{q}}{D t}+\mathfrak{q} \circledast \mathfrak{q}+\mathfrak{q}_{p}=0,
$$

которое можно записать явно в переменных $\alpha, \boldsymbol{\chi}$ :

$$
\frac{D \alpha}{D t}+\alpha^{2}-\chi^{2}+\alpha_{p}=0, \quad \frac{D \chi}{D t}+2 \alpha \chi+\chi_{p}=0 .
$$

В теореме 1 для вычисления траектории ортонормированного кватернионного репера $(\widehat{\boldsymbol{\omega}}, \widehat{\boldsymbol{\chi}}, \widehat{\boldsymbol{\omega}} \times \widehat{\boldsymbol{\chi}})$ нам понадобился вектор $\boldsymbol{b}=-P \boldsymbol{\omega}$. Точнее, необходимо решить уравнения

$$
\begin{aligned}
\frac{D \widehat{\boldsymbol{\omega}}}{D t} & =\mathcal{D} \times \widehat{\boldsymbol{\omega}}, \\
\frac{D(\widehat{\boldsymbol{\omega}} \times \widehat{\boldsymbol{\chi}})}{D t} & =\mathcal{D} \times(\widehat{\boldsymbol{\omega}} \times \widehat{\boldsymbol{\chi}}), \\
\frac{D \widehat{\chi}}{D t} & =\mathcal{D} \times \widehat{\chi},
\end{aligned}
$$

где вектор угловой скорости Дарбу $\mathcal{D}$ определен в виде

$$
\mathcal{D}=\chi+\frac{c_{p}}{\chi} \widehat{\boldsymbol{\omega}}, \quad c_{p}=-\widehat{\boldsymbol{\omega}} \cdot\left(\widehat{\chi} \times \chi_{p}\right) .
$$

Гессиан давления участвует в угловой скорости $\mathcal{D}$ через посредство скалярного коэффициента $c_{p}$. Чтобы вычислить траектории материальных фрагментов течения, необходимы данные о гессиане давления $P$, а также о вихре $\boldsymbol{\omega}$ и о матрице деформации $S$. В этом месте явно обнаруживается принципиальное различие между уравнениями Эйлера и подчиненной проблемой. Для уравнений Эйлера поле $\boldsymbol{b}$, содержащее $P$, не является независимым от $\boldsymbol{w} \equiv \boldsymbol{\omega}$, но

\footnotetext{
${ }^{6} \mathrm{~B}$ работах [55], [56], [66], [67] единичный вектор вихря обозначался $\xi$, а здесь мы его обозначаем $\widehat{\boldsymbol{\omega}}$.

${ }^{7}$ В принципе уравнение (3.9) можно линеаризовать к уравнению Шрёдингера для нулевого собственного значения с потенциалом $\mathfrak{q}_{p}$, но неясно, как действовать дальше.
} 


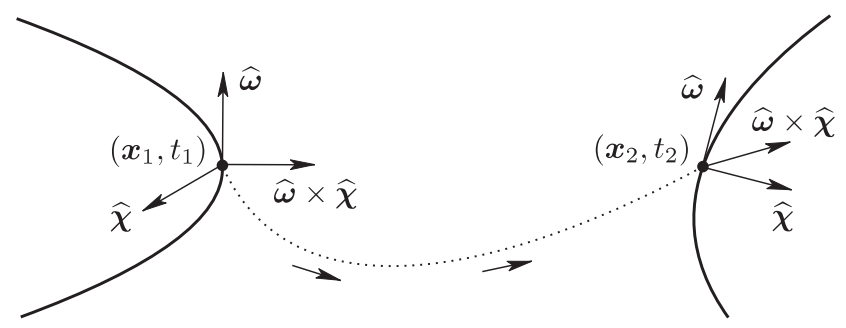

Рис. 2. Это изображение аналогично рис. 1, но пунктирная линия здесь обозначает траекторию фрагмента $(\bullet)$ эйлерова течения, который перемещается из точки $\left(\boldsymbol{x}_{1}, t_{1}\right)$ в точку $\left(\boldsymbol{x}_{2}, t_{2}\right)$. Сплошные линии представляют вихревые линии, для которых $\widehat{\omega}$ является единичным вектором нормали. Ориентация кватернионного репера $(\widehat{\boldsymbol{\omega}}, \widehat{\boldsymbol{\chi}}, \widehat{\boldsymbol{\omega}} \times \widehat{\chi})$ показана для двух пространственно-временных точек; отметим, что он не совпадает с репером Френе, отвечающим траектории данного фрагмента.

они связаны почти неуловимо и нелокально через эллиптическое уравнение для давления (1.5), которое мы здесь повторяем,

$$
-\operatorname{Tr} P=\operatorname{Tr} S^{2}-\frac{1}{2} \omega^{2}
$$

Теорема 2 выражает эволюцию $\mathfrak{q}_{p}$ уравнением

$$
\frac{D \mathfrak{q}_{p}}{D t}=\mathfrak{q} \circledast \mathfrak{q}_{p}+\lambda_{1} \mathfrak{q}_{p}-\lambda_{2} \mathfrak{q}-\lambda_{3} \mathbb{I}
$$

в терминах произвольных скаляров $\lambda_{i}(\boldsymbol{x}, t)$. Однако как их находить, используя условие несжимаемости, пока неясно.

\section{4. Теорема ВКМ и направление вихря}

Решения трехмерного уравнения Эйлера могут быстро становиться негладкими, и поэтому вопросы о том, как и в каком направлении растет вихрь, очень важны, хотя пока еще и остаются без определенного ответа. Понятно, что растяжение вихря $\boldsymbol{\omega} \cdot \nabla \boldsymbol{u}=S \boldsymbol{\omega}$ и выравнивание $\boldsymbol{\omega}$ по собственным векторам $\boldsymbol{e}_{i}$ матрицы $S$ играют фундаментальную роль при формировании сингулярности за конечное время. Основные результаты компьютерного моделирования этого явления можно найти в работах [68]-[76]. Изучение сингулярностей в комплексном расширении времени для двумерных уравнений Эйлера имеется в [77], где также дается обширная литература по этому вопросу.

Теорема BКМ [52] является ключевым результатом при изучении роста эйлерова вихря и возможного сингулярного поведения. Область $\mathbb{D} \subset \mathbb{R}^{3}$ в теореме 3 выбрана периодической в демонстрационных целях, чтобы гарантировать локальное (по времени) существование классических решений [78], хотя она применима и к более общим областям. Сформулируем один из вариантов этой теоремы $\left(H^{s}\right.$ обозначает стандартное пространство Соболева). 
Теорема 3 [52]. В области $\mathbb{D}=[0, L]_{\mathrm{per}}^{3}$ существует глобалъное решение уравнений Эйлера $\boldsymbol{u} \in C\left([0, \infty] ; H^{s}\right) \cap C^{1}\left([0, \infty] ; H^{s-1}\right)$ nри $s \geqslant 3$, если для любого $T>0$

$$
\int_{0}^{T}\|\boldsymbol{\omega}\|_{L^{\infty}(\mathbb{D})} d \tau<\infty .
$$

Этот результат можно также сформулировать обратным образом: сингулярность не может образоваться к моменту $T$ без условия, что $\int_{0}^{T}\|\boldsymbol{\omega}\|_{L^{\infty}(\mathbb{D})} d \tau=\infty$. Теорема 3 имеет прямые вычислительные следствия. В гипотетическом компьютерном эксперименте, если обнаруживается рост вихря порядка $\|\boldsymbol{\omega}\|_{L^{\infty}(\mathbb{D})} \sim$ $(T-t)^{-\gamma}$ при некотором $\gamma>0$, то теорема утверждает, что $\gamma$ обязано удовлетворять неравенству $\gamma \geqslant 1$ для наблюдаемого реального (или нет) сингулярного поведения артефакта численных вычислений. Причина состоит в том, что если $\gamma$ не лежит в интервале $0<\gamma<1$, то $\|\boldsymbol{\omega}\|_{L^{\infty}(\mathbb{D})}$ взрывается, в то время как для его интеграла по времени этого не происходит, что противоречит теореме. На основе многих численных экспериментов с уравнениями Эйлера, проведенных в [71], [72] с использованием антипараллельных вихревых трубок в качестве начальных данных, было впервые обнаружено, что $\gamma$ проходит порог с критическим значением $\gamma=1$. Затем последовали работы [73]-[75]. Недавние численные результаты полученные в [76], противоречат, однако, существованию сингулярности: см. обсуждение этих вопросов в [79]. Чтобы полностью определиться с этой проблемой, потребуются более тщательные вычислительные эксперименты в сочетании с анализом той роли, которую играет направление роста вихря. Как указано в разделе 1, работа [56] (см. также [55]) была первой, в которой приведена точная математическая формулировка того, насколько гладко должно быть направление вихревых линий, чтобы привести к возникновению сингулярности или, наоборот, предупредить ее образование. Пункт 4.1 посвящен краткому обзору этой работы. Более поздние работы [80], [57], [58] и [66], [67] являются вариациями на ту же тему. Подход, предложенный в пионерской работе [56], лежит в основе компьютерных экспериментов последующего поколения по методу [71], [72], [79] и [76] для проверки того, происходит ли развитие особенности. Пункт 4.2 посвящен представлению результатов работ [57], [58], в которых был установлен более слабый критерий для вихревых линий. В п. 4.3 излагается альтернативная теорема о направлении вихря, доказанная в [59] на основе кватернионного формализма.

Ссылки и дальнейшие перспективы при изучении уравнений Эйлера можно найти в книге [15]. В [81] построены очень слабые решения, которые имеют определенные реалистические свойства, но их кинетическая энергия монотонно убывает по времени, и они являются всюду разрывными и неограниченными; об их динамике в более экзотических пространствах см. [82]-[85].

4.1. Работы Константина, Феффермана и Майды. Очевидный вопрос относительно критерия ВКМ связан с возможностью ослабления нормы $L^{\infty}$ до $L^{p}$ при некотором $1 \leqslant p \leqslant \infty$. Эта проблема рассматривалась в работе П. Константина [55], где делались дальнейшие предположения о локальной природе векторных полей вихря и скорости. Рассмотрим поле скорости

$$
U_{1}(t):=\sup _{\boldsymbol{x}}|\boldsymbol{u}(\boldsymbol{x}, t)|
$$


и $L_{\mathrm{loc}}^{1}$-норму $\boldsymbol{\omega}$, определенную по формуле

$$
\|\boldsymbol{\omega}\|_{1, \text { loc }}=L^{-3} \sup _{\boldsymbol{x}} \int_{|\boldsymbol{y}| \leqslant L}|\boldsymbol{\omega}(\boldsymbol{x}+\boldsymbol{y})| d^{3} y,
$$

где $L$ - некоторый внешний характерный размер (масштаб длины) течения Эйлера, который можно принять за единицу. Предположим теперь, что единичный вектор вихря является липшицевым, т.е.

$$
|\widehat{\boldsymbol{\omega}}(\boldsymbol{x}, t)-\widehat{\boldsymbol{\omega}}(\boldsymbol{y}, t)| \leqslant \frac{|\boldsymbol{x}-\boldsymbol{y}|}{\rho_{0}(t)}
$$

при $|\boldsymbol{x}-\boldsymbol{y}| \leqslant L$ для некоторой длины $\rho_{0}(t)$. В работе [55] сформулирован следующий результат, который затем был переформулирован и доказан в работе П. Константина, Ч. Феффермана и Э. Майды [56].

ТЕОРема 4 [55], [56]. Пусть начальный вихрь $\boldsymbol{\omega}_{0}$ является гладким и имеет компактный носитель, и пусть решение уравнений Эйлера удовлетворяет неравенствам

$$
\int_{0}^{T}\|\boldsymbol{\omega}(\cdot, s)\|_{1, \text { loc }}\left(\frac{L}{\rho_{0}(s)}\right)^{3} d s<\infty, \quad \int_{0}^{T} \frac{U(s)}{\rho_{0}(s)} d s<\infty .
$$

Тогда

$$
\sup _{0 \leqslant t \leqslant T} \frac{\|\boldsymbol{\omega}(\cdot, t)\|_{\infty}}{\|\boldsymbol{\omega}(\cdot, t)\|_{1, \text { loc }}}<\infty
$$

Ясно, что если $U_{1}=\|\boldsymbol{u}\|_{\infty}<\infty$ и $\|\boldsymbol{\omega}(\cdot, t)\|_{1, \text { loc }}<\infty$ на отрезке $[0, T]$, а функция $\rho_{0}$ отделена от нуля, то в теореме ВКМ говорится о невозможности появления сингулярности. Условие Липшица (4.4) можно сформулировать иначе, если рассматривать антипаралельные вихревые трубки [55].

Затем в работе [56] более подробно поднимается вопрос об определении понятия "гладкой направленности" для траекторий. Рассмотрим трехмерные уравнения Эйлера с гладкими локализованными начальными данными; предположим, что их решение является гладким на интервале $0 \leqslant t<T$. Поле скоростей определяет траектории фрагментов $\boldsymbol{X}\left(\boldsymbol{x}_{0}, t\right)$, которые удовлетворяют

$$
\frac{D \boldsymbol{X}}{D t}=\boldsymbol{u}(\boldsymbol{X}, t), \quad \boldsymbol{X}\left(\boldsymbol{x}_{0}, 0\right)=\boldsymbol{x}_{0} .
$$

Образ $\boldsymbol{W}_{t}$ множества $\boldsymbol{W}_{0}$ задается формулой $\boldsymbol{W}_{t}=\boldsymbol{X}\left(t, \boldsymbol{W}_{0}\right)$. Тогда множество $W_{0}$ называется гладко направленным, если найдутся длина $\rho>0$ и радиус шара $0<r<\frac{1}{2} \rho$ такие, что выполнены следующие три условия.

1. Для каждого $\boldsymbol{x}_{0} \in \boldsymbol{W}_{0}^{*}$, где $\boldsymbol{W}_{0}^{*}=\left\{\boldsymbol{x}_{0} \in \boldsymbol{W}_{0}^{*} ;\left|\boldsymbol{\omega}_{0}\left(\boldsymbol{x}_{0}\right)\right| \neq 0\right\}$, и для всех $t \in[0, T)$ функция $\widehat{\boldsymbol{\omega}}(\cdot, t)$ имеет липшицево продолжение на шар $B_{4 \rho}$ радиуса $4 \rho$ с центром $\boldsymbol{X}\left(\boldsymbol{x}_{0}, t\right)$, причем

$$
M=\lim _{t \rightarrow T} \sup _{\boldsymbol{x}_{0} \in \boldsymbol{W}_{0}^{*}} \int_{0}^{t}\|\nabla \widehat{\boldsymbol{\omega}}(\cdot, s)\|_{L^{\infty}\left(B_{4 \rho}\right)}^{2} d s<\infty .
$$

Это условие гарантирует, что направление вихря хорошо ведет себя в окрестности множества траекторий. 
2. Неравенство

$$
\sup _{B_{3 r}\left(\boldsymbol{W}_{t}\right)}|\boldsymbol{\omega}(\boldsymbol{x}, t)| \leqslant m \sup _{B_{r}\left(\boldsymbol{W}_{t}\right)}|\boldsymbol{\omega}(\boldsymbol{x}, t)|
$$

выполнено для всех $t \in[0, T)$ при некоторой константе $m=$ const $>0$. Это просто означает, что выбранная окрестность захватывает большой и растущий вихрь, но она не настолько большая, чтобы перекрываться с другими аналогичными областями.

3. Поле скоростей в шаре радиуса $4 \rho$ удовлетворяет неравенству

$$
\sup _{B_{4 r}\left(\boldsymbol{W}_{t}\right)}|\boldsymbol{u}(\boldsymbol{x}, t)| \leqslant U(t):=\sup _{\boldsymbol{x}}|\boldsymbol{u}(\boldsymbol{x}, t)|<\infty
$$

при всех $t \in[0, T)$.

Теорема 5 [56]. Предположим, что $\boldsymbol{W}_{0}$ является гладко направленным в соответствии с данным выше определением 1-3. Тогда имеются момент времени $\tau>0$ и константа $\Gamma$ такие, что неравенство

$$
\sup _{B_{r}\left(\boldsymbol{W}_{t}\right)}|\boldsymbol{\omega}(\boldsymbol{x}, t)| \leqslant \Gamma \sup _{B_{\rho}\left(\boldsymbol{W}_{t}\right)}\left|\boldsymbol{\omega}\left(\boldsymbol{x}, t_{0}\right)\right|
$$

выполнено при всех $0 \leqslant t_{0}<T u 0 \leqslant t-t_{0} \leqslant \tau$.

Условие 2 может иметь импликацию о том, насколько естественная длина $\rho$ масштабирует время развития потока [72], однако, чтобы понять это, требуется дополнительная работа. В работе [80] условие 3 ослаблено для случая вихревых трубок до неравенства

$$
\int_{0}^{T} U(s) d s=\int_{0}^{T}\|\boldsymbol{u}(\cdot, s)\|_{\infty} d s<\infty .
$$

4.2. Работы Дена, Хоу и Ю. В работе Дена, Хоу и Ю [57] первый и, возможно, самый важный критерий “гладкой направленности", а именно, неравенство (4.8), был переработан от локального контроля посредством интеграла $\int_{0}^{t}\|\nabla \widehat{\boldsymbol{\omega}}(\cdot, t)\|_{L^{\infty}}^{2} d t$ по $0 \leqslant t \leqslant T$ до условия на длину дуги $s$ (натуральный параметр) между двумя точками $s_{1}$ и $s_{2}$. Первый из доказанных ими результатов формулируется следующим образом.

TЕОРема 6 [57]. Пусть $\boldsymbol{x}(t)$ обозначает семейство точек таких, что $|\boldsymbol{\omega}(\boldsymbol{x}(t), t)| \gtrsim \Omega(t):=\|\boldsymbol{\omega}\|_{\infty}$. Предположим, что для любого $t \in[0, T]$ найдется другая точка $\boldsymbol{y}(t)$ на той же вихревой линии, что и $\boldsymbol{x}(t)$, такая, что единичный вектор вихря $\widehat{\boldsymbol{\omega}}(\boldsymbol{x}, t)$ вдоль линии между $\boldsymbol{x}(t)$ и $\boldsymbol{y}(t)$ корректно задан. Если дополнительно предположить, что

$$
\left|\int_{s_{1}}^{s_{2}} \operatorname{div} \widehat{\boldsymbol{\omega}}(s, t) d s\right| \leqslant C(T)
$$

u, кроме того,

$$
\int_{0}^{T}|\boldsymbol{\omega}(\boldsymbol{x}(t), t)| d t<\infty
$$

то вплоть до момента времени Т взрыва решения не произойдет. Более того,

$$
e^{-C} \leqslant \frac{|\boldsymbol{\omega}(\boldsymbol{x}(t), t)|}{|\boldsymbol{\omega}(\boldsymbol{y}(t), t)|} \leqslant e^{C} .
$$


Неравенство (4.13) основано на простом факте, что

$$
0=\operatorname{div} \boldsymbol{\omega}=|\boldsymbol{\omega}| \operatorname{div} \widehat{\boldsymbol{\omega}}+\widehat{\boldsymbol{\omega}} \cdot \nabla|\boldsymbol{\omega}|=|\boldsymbol{\omega}| \operatorname{div} \widehat{\boldsymbol{\omega}}+\frac{d|\boldsymbol{\omega}|}{d s},
$$

где $\widehat{\boldsymbol{\omega}} \cdot \nabla=\frac{d}{d s}-$ производная по натуральному параметру.

Второй, даже более важный, результат Дена, Хоу и Ю [58] основан на рассмотрении семейства сегментов вихревой линии $L_{t}$, вдоль которой максимальный вихрь сравним с максимальным вихрем $\Omega(t)$. Пусть $L(t)$ обозначает длину дуги $L_{t}, \hat{\boldsymbol{n}}$ - единичный вектор нормали и $\kappa$ - кривизну вихревой линии. Определим величины

$$
\begin{aligned}
U_{\widehat{\boldsymbol{\omega}}}(t) & \equiv \max _{\boldsymbol{x}, \boldsymbol{y} \in L_{t}}|(\boldsymbol{u} \cdot \widehat{\boldsymbol{\omega}})(\boldsymbol{x}, t)-(\boldsymbol{u} \cdot \widehat{\boldsymbol{\omega}})(\boldsymbol{y}, t)|, \\
U_{n}(t) & \equiv \max _{L_{t}}|\boldsymbol{u} \cdot \hat{\boldsymbol{n}}|
\end{aligned}
$$

И

$$
M(t) \equiv \max \left(\|\nabla \cdot \widehat{\boldsymbol{\omega}}\|_{L^{\infty}\left(L_{t}\right)},\|\kappa\|_{L^{\infty}\left(L_{t}\right)}\right) .
$$

Теорема 7 [57], [58]. Пусть $A, B \in(0,1)$, причем $B<1-A, u C_{0}$ - некоторая положительная константа. Если

1) $U_{\widehat{\omega}}(t)+U_{n}(t) \lesssim(T-t)^{-A}$,

2) $M(t) L(t) \leqslant C_{0}$,

3) $L(t) \gtrsim(T-t)^{B}$, то взрыва не будет до момента времени $T$.

В работе [58] неравенство $A+B<1$ было заменено на равенство $A+B=1$, а также даны другие ослабленные условия. Кроме того, там также выведено некоторое геометрическое условие типа масштаба, которое можно применять, когда скорость взрывается одновременно с вихрем и коэффициент взрыва скорости пропорционален квадратному корню из вихря. В этом заключается наихудший сценарий взрыва поля скорости в соответствии с теоремой Кельвина о циркуляции.

4.3. Непостоянство $\alpha_{p}$ и $\chi_{p}$ : кватернионы и направление вихря. Ключевым соотношением при использовании кватернионного формализма в уравнениях Эйлера является уравнение Риккати $(3.9)$ для $\mathfrak{q}=[\alpha(x, t), \chi(x, t)]$. В терминах переменных $\alpha$ и $\chi$ оно распадается на четыре уравнения

$$
\frac{D \alpha}{D t}=\chi^{2}-\alpha^{2}-\alpha_{p}, \quad \frac{D \chi}{D t}=-2 \alpha \chi-\chi_{p} .
$$

Хотя дифференциальные уравнения для $\mathfrak{q}_{p}=\left[\alpha_{p}, \chi_{p}\right]$ выглядят просто, ясно, что $\mathfrak{q}_{p}$ зависит от решения, так как имеется ограничение на давление: $-\operatorname{Tr} P=u_{i, k} u_{k, i}$. Поэтому заманчиво считать, что $\mathfrak{q}_{p}$ ведет себя подобно константе; и это может оказаться справедливым для больших областей эйлеровых потоков, однако это точно не верно для областей с наиболее интенсивными завихрениями, где вихревые линии имеют наибольшую кривизну. В таких областях знаки $\alpha_{p}$ и компонент $\boldsymbol{\chi}_{p}$ могут меняться весьма непредсказуемым образом [64]. В силу этих потенциально резких изменений, кватернион $\mathfrak{q}_{p}$ можно рассматривать в качестве кандидата для формулирования в его терминах новых условий в теоремах о вихре в развитие темы из пп. 4.1-4.2. Другие ограничения на $P$ представлены в работе Д. Чаэ [67]. 
В [56]-[58] показано, что функцию $\nabla \boldsymbol{w}$ необходимо как-то контролировать в локальных областях, в которых линии вихря имеют большую кривизну. В терминах числа производных гессиан $P$ находится на том же уровне, и мы будем искать контроль решений уравнений Эйлера именно в терминах $P$ и связанных с ним переменных $\alpha_{p}$ и $\chi_{p}$. Из определения легко проверить, что $\alpha^{2}+\chi^{2}=|S \widehat{\boldsymbol{\omega}}|^{2}$, и поэтому на линиях вихря $\alpha=\alpha\left(\boldsymbol{X}\left(t, \boldsymbol{x}_{0}\right), t\right)$ система $(4.20)$ становится

$$
\frac{d}{d t}|S \widehat{\boldsymbol{\omega}}|^{2}=-\alpha|S \widehat{\boldsymbol{\omega}}|^{2}+\alpha \alpha_{p}+\boldsymbol{\chi} \cdot \boldsymbol{\chi}_{p}
$$

После интегрирования получаем

$$
|S \widehat{\boldsymbol{\omega}}(\boldsymbol{X}(\tau), t)|^{2}=-2 \int_{0}^{T} e^{\int_{0}^{\tau} \alpha\left(\cdot, t^{\prime}\right) d t^{\prime}-\int_{0}^{t} \alpha\left(\cdot, t^{\prime}\right) d t^{\prime}}\left(\alpha \alpha_{p}+\boldsymbol{\chi} \cdot \boldsymbol{\chi}_{p}\right) \boldsymbol{X}(\cdot, \tau) d \tau .
$$

Теперь имеются две возможности. Первая состоит в применении неравенства Коши-Шварца и использовании равенства $\alpha_{p}^{2}+\chi_{p}^{2}=|P \widehat{\boldsymbol{\omega}}|^{2}$ :

$$
\left|S \widehat{\boldsymbol{\omega}}\left(\boldsymbol{X}\left(t, \boldsymbol{x}_{0}\right), t\right)\right| \leqslant 2 \int_{0}^{T} e^{\int_{0}^{\tau} \alpha\left(\cdot, t^{\prime}\right) d t^{\prime}-\int_{0}^{t} \alpha\left(\cdot, t^{\prime}\right) d t^{\prime}}|P \widehat{\boldsymbol{\omega}}(\cdot, \tau)| d \tau .
$$

Этот путь аналогичен подходу Чаэ (теорема 5.1 в [67]), основанному на контролировании интеграла по времени от $\|S \widehat{\boldsymbol{\omega}} \cdot P \widehat{\boldsymbol{\omega}}\|_{\infty}$, который выводится из (3.2).

Вторая возможность выявляет интересный случай исследования направления вихря с использованием $\chi_{p}$. Ее можно также рассматривать как альтернативный путь для изучения направления вихря после работ [56]-[58]. Вектор $\chi_{p}=\widehat{\boldsymbol{\omega}} \times P \widehat{\boldsymbol{\omega}}$ содержит $\widehat{\boldsymbol{\omega}}$, а не $\boldsymbol{\omega}$, и поэтому он связан с направлением $\boldsymbol{\omega}$, а не с его модулем. Прежде всего мы используем тот факт, что $|\boldsymbol{\omega}|$ не может взорваться при $\alpha<0$, поскольку $D|\boldsymbol{\omega}| / D t=\alpha|\boldsymbol{\omega}|$; значит, следует рассмотреть $\alpha \geqslant 0$. В случае, когда угол между $\widehat{\boldsymbol{\omega}}$ и $P \widehat{\boldsymbol{\omega}}$ не равен нулю,

$$
\left|S \widehat{\boldsymbol{\omega}}\left(\boldsymbol{X}\left(t, \boldsymbol{x}_{0}\right), t\right)\right| \leqslant 2 \int_{0}^{T}\left|\chi_{p}(\cdot, \tau)\right| d \tau .
$$

Если правая часть ограничена, то решение уравнения Эйлера не может взорваться, за исключением возможности, когда $|P \widehat{\boldsymbol{\omega}}|$ взрывается одновременно с тем, что угол между $\widehat{\boldsymbol{\omega}}$ и $P \widehat{\boldsymbol{\omega}}$ стремится к нулю, а $\boldsymbol{\chi}_{p}$ остается конечной. При таких обстоятельствах $\int_{0}^{t}\left|\chi_{p}\right| d \tau<\infty$, но $\int_{0}^{t}\left|\alpha_{p}\right| d \tau \rightarrow \infty$, и, значит, в этом случае взрыв теоретически возможен. Этот результат не гарантирует, что взрыв обязательно случится при выполнении условия коллинеарности; это просто означает, что при выполнении (4.24) это событие возможно. Указанная коллинеарность была замечена в работах [36] и [40]; там было также обнаружено с помощью численного анализа, что в точках максимума энстрофии вектор $\boldsymbol{\omega}$ стремится выстроиться вдоль собственных векторов матрицы $P$, отвечающих самым отрицательным ее собственным значениям. После выражения по всему периодическому объему получается следующая теорема.

ТеОрема 8 [59]. В области $\mathbb{D}=[0, L]_{\mathrm{per}}^{3}$ существует глобальное решение уравнений Эйлера $\boldsymbol{u} \in C\left([0, \infty] ; H^{s}\right) \cap C^{1}\left([0, \infty] ; H^{s-1}\right)$ при $s \geqslant 3$, если для каждого $T>0$

$$
\int_{0}^{T}\left\|\chi_{p}\right\|_{L^{\infty}(\mathbb{D})} d \tau<\infty
$$

за исключением случая, когда $\widehat{\boldsymbol{\omega}}$ становится коллинеарным собственному вектору функиии $P$ в момент времени $T$. 


\section{5. Завершающий пример: уравнения несжимаемой идеальной МГД}

Лагранжев формализм, описанный в разделе 2, может быть применен ко многим ситуациям, например растяжению элементов-линий жидкости, несжимаемому движению эйлеровых жидкостей и идеальной МГД (см. [15]). В качестве завершающего примера мы выбрали идеальную МГД в форме с переменными Элзассера; другой подход изложен в [86]. Уравнения жидкости в магнитном поле $\boldsymbol{B}$ имеют вид

$$
\begin{aligned}
\frac{D \boldsymbol{u}}{D t} & =\boldsymbol{B} \cdot \nabla \boldsymbol{B}-\nabla p, \\
\frac{D \boldsymbol{B}}{D t} & =\boldsymbol{B} \cdot \nabla \boldsymbol{u}
\end{aligned}
$$

с условиями $\operatorname{div} \boldsymbol{u}=0$ и $\operatorname{div} \boldsymbol{B}=0$. Давление $p$ в (5.1) равно $p_{f}+\frac{1}{2} B^{2}$, где $p_{f}-$ давление жидкости. Переменные Элзассера определяются в виде следующей линейной комбинации [30]:

$$
\boldsymbol{v}^{ \pm}=\boldsymbol{u} \pm \boldsymbol{B}
$$

Существование двух скоростей $\boldsymbol{v}^{ \pm}$означает наличие двух материальных производных

$$
\frac{D^{ \pm}}{D t}=\frac{\partial}{\partial t}+\boldsymbol{v}^{ \pm} \cdot \nabla
$$

Используя эти обозначения, уравнения (5.1) и (5.2) можно переписать в виде

$$
\frac{D^{ \pm} \boldsymbol{v}^{\mp}}{D t}=-\nabla p
$$

где магнитное поле $\boldsymbol{B}$ удовлетворяет $\left(\operatorname{div} \boldsymbol{v}^{ \pm}=0\right)$

$$
\frac{D^{ \pm} \boldsymbol{B}}{D t}=\boldsymbol{B} \cdot \nabla \boldsymbol{v}^{ \pm}
$$

Следовательно, имеется пара троек $\left(\boldsymbol{v}^{ \pm}, \boldsymbol{B}, \boldsymbol{a}^{ \pm}\right)$, где функции $\boldsymbol{a}^{ \pm}=\boldsymbol{B} \cdot \nabla \boldsymbol{v}^{ \pm}$ построены на основе предложенной Г. К. Моффатом интерпретации $\boldsymbol{B}$-поля как важного элемента растяжения [30]. Из [65], [59] имеем

$$
\frac{D^{ \pm} \boldsymbol{a}^{\mp}}{D t}=-P \boldsymbol{B}
$$

где $\boldsymbol{b}^{ \pm}=-P \boldsymbol{B}$. Имея два квартета $\left(\boldsymbol{v}^{ \pm}, \boldsymbol{B}, \boldsymbol{a}^{ \pm}, \boldsymbol{b}\right)$, из результатов раздела 2 получаем для двух лагранжевых производных два уравнения Риккати

$$
\frac{D^{\mp} \mathfrak{q}_{a}^{ \pm}}{D t}+\mathfrak{q}_{a}^{ \pm} \circledast \mathfrak{q}_{a}^{\mp}=\mathfrak{q}_{b} .
$$

В итоге, кватернионную МГД необходимо интерпретировать в терминах двух множеств ортонормированных реперов $\left(\widehat{\boldsymbol{B}}, \widehat{\boldsymbol{\chi}}^{ \pm}, \widehat{\boldsymbol{B}} \times \widehat{\boldsymbol{\chi}}^{ \pm}\right)$, к которым применяются операторы противоположных лагранжевых производных по времени:

$$
\begin{aligned}
\frac{D^{\mp} \widehat{\boldsymbol{B}}}{D t} & =\mathcal{D}^{\mp} \times \widehat{\boldsymbol{B}}, \\
\frac{D^{\mp}}{D t}\left(\widehat{\boldsymbol{B}} \times \widehat{\boldsymbol{\chi}}^{ \pm}\right) & =\mathcal{D}^{\mp} \times\left(\widehat{\boldsymbol{B}} \times \widehat{\boldsymbol{\chi}}^{ \pm}\right), \\
\frac{D^{\mp} \widehat{\chi}^{ \pm}}{D t} & =\mathcal{D}^{\mp} \times \widehat{\boldsymbol{\chi}}^{ \pm},
\end{aligned}
$$


где пара векторов Дарбу-Элзассера $\mathcal{D}^{\mp}$ определяется по формулам

$$
\mathcal{D}^{\mp}=\chi^{\mp}-\frac{c_{B}^{\mp}}{\chi^{\mp}} \widehat{\boldsymbol{B}}, \quad c_{B}^{\mp}=\widehat{\boldsymbol{B}} \cdot\left[\widehat{\chi}^{ \pm} \times\left(\chi_{p B}+\alpha^{ \pm} \chi^{\mp}\right)\right] .
$$

\section{6. Заключение}

Успешное применение кватернионов в аэрокосмической промышленности и в компьютерной анимации, которое было когда-то предвосхищено Гамильтоном, дает надежную гарантию и уверенность, что кватернионы также применимы к описанию “летающих" лагранжевых фрагментов, которые в равной степени могут служить пассивно переносимыми метками и, в особенности, материальными фрагментами трехмерных эйлеровых течений. Эквивалентная формулировка для сжимаемых уравнений Эйлера [46], [47] может дать ключ к пониманию природы несжимаемого предела. Эта тема будет обсуждаться в готовящейся к публикации статье автора с Г. Эсраги. Случай баротропных сжимаемых уравнений Эйлера и другие примеры объединены в табл. 2.

ТАБлицА 2. Различные примеры применения теоремы Эртеля для замыкания квартета векторов $(\boldsymbol{u}, \boldsymbol{w}, \boldsymbol{a}, \boldsymbol{b})$. Для идеальной МГД производные $D^{ \pm} / D t$ определены в $(5.4)$.

\begin{tabular}{c|c|c|c|c|c} 
Система & $\boldsymbol{u}$ & $\boldsymbol{w}$ & $\boldsymbol{a}$ & $\boldsymbol{b}$ & $\begin{array}{c}\text { Материальная } \\
\text { производная }\end{array}$ \\
\hline $\begin{array}{c}\text { несжимаемый } \\
\text { Эйлер }\end{array}$ & $\boldsymbol{u}$ & $\boldsymbol{x}$ & $\boldsymbol{u}$ & $-\boldsymbol{\nabla} p$ & $D / D t$ \\
$\begin{array}{c}\text { несжимаемый } \\
\text { Эйлер }\end{array}$ & $\boldsymbol{u}$ & $\boldsymbol{\omega}$ & $S \boldsymbol{\omega}$ & $-P \boldsymbol{\omega}$ & $D / D t$ \\
$\begin{array}{c}\text { баротропный } \\
\text { Эйлер }\end{array}$ & $\boldsymbol{u}$ & $\boldsymbol{\omega} / \rho$ & $\boldsymbol{\omega} / \rho \cdot \boldsymbol{\nabla} \boldsymbol{u}$ & $-\left(\omega_{j} / \rho\right) \partial_{j}\left(\rho \partial_{j} p\right)$ & $D / D t$ \\
МГД & $\boldsymbol{v}^{ \pm}$ & $\boldsymbol{B}$ & $\boldsymbol{B} \cdot \boldsymbol{\nabla} \boldsymbol{v}^{\mp}$ & $-P \boldsymbol{B}$ & $D^{ \pm} / D t$ \\
Смесь & $\boldsymbol{u}$ & $\boldsymbol{\delta} \boldsymbol{\ell}$ & $\boldsymbol{\delta} \boldsymbol{\ell} \cdot \boldsymbol{\nabla} \boldsymbol{u}$ & $-P \boldsymbol{\delta} \boldsymbol{D}$ & $D / D t$
\end{tabular}

Там, где логично и естественно возникают кватернионы, система обладает определенными геометрическими свойствами. Для уравнений Эйлера знаменательным является тот факт, что кватерионы выявляют коэффициент роста $\alpha$ и частоту вращения $\chi$ вектора вихря, причем последняя весьма чувствительна к направлению вихря по отношению к собственным векторам $S$. Чтобы увидеть это, рассмотрим вихрь Бюргерса, который представляет собой вихревую трубку [18]. Собственный вектор $S$ направлен вдоль оси трубки параллельно $\boldsymbol{\omega}$, и в этом случае $\chi=\widehat{\boldsymbol{\omega}} \times S \widehat{\boldsymbol{\omega}}=0$. Однако если трубка подходит близко к другой трубке, то они начинают изгибаться и могут переплестись. Как только кривизна трубки становится ненулевой вдоль некоторого участка линии, то сразу $\chi \neq 0$ вдоль этого же участка. Подобным же образом это происходит в вихрях со слоями, которые изгибаются или сворачиваются, приближаясь к другому слою. Следовательно, трехмерный вектор $\chi$ весьма чувствителен к топологии вихря и локально зависим от нее. В каждой точке его эволюция самым элегантным образом выражается с помощью ассоциированного кватерниона q, 
который обязан удовлетворять уравнению (см. (3.9))

$$
\frac{D \mathfrak{q}}{D t}+\mathfrak{q} \circledast \mathfrak{q}+\mathfrak{q}_{p}=0 .
$$

Чтобы полностью оценить силу метода, обратим внимание на то, что поле давления должно с необходимостью возникнуть явно в форме своего гессиана посредством $\mathfrak{q}_{p}$, однако это противоречит общепризнанной практике при изучении динамики жидкости, когда давление исключается посредством оператора Лерэ. Гессиан давления появляется в материальной производной члена растяжения вихря, через использование теоремы Эртеля, в качестве платы за устранение нелинейности $O\left(|\boldsymbol{\omega}||\nabla \boldsymbol{u}|^{2}\right)$. На самом деле, влияние гессиана давления на член растяжения вихря является едва уловимым и нелокальным. Поэтому, хоть и заманчиво отбросить давление, раз оно исчезает из уравнения вихря, завуалированно оно может оказаться одним из самых важных членов в динамике невязкой жидкости.

Разумеется, уравнение (6.1) имеет стационарные решения, и одно из них есть $\chi=\chi_{p}=0$, где $\alpha=\alpha_{0}$ и $\alpha_{p}=-\alpha_{0}^{2}$. Вихрь Бюргерса относится к решениям этого типа см. [64], [65]. Уделив особое внимание в п. 4.3 непостоянству $\alpha_{p}$ и $\chi_{p}$ для интенсивных и потенциально сингулярных областей, постараемся теперь выявить простейшее общие черты в поведении величин $\alpha$ и $\chi$ из (4.20), когда $\alpha_{p}$ и $\chi_{p}$ являются постоянными, например, для вихря, близкого к вихрю Бюргерса. Для этого рассмотрим четыре уравнения, которые получаются из (6.1) наподобие (4.20), и будем их считать обыкновенными дифференциальными уравнениями на траекториях фрагментов $\boldsymbol{X}\left(t, \boldsymbol{x}_{0}\right)$ :

$$
\dot{\alpha}=\chi^{2}-\alpha^{2}-\alpha_{p}, \quad \dot{\chi}=-2 \alpha \chi-C_{p}
$$

На фазовой плоскости $\alpha, \chi$, где $\alpha_{p}=$ const и $C_{p}=\widehat{\chi} \cdot \chi_{p}=$ const, имеются две критические точки

$$
(\alpha, \chi)=\left( \pm \alpha_{0}, \chi_{0}\right), \quad 2 \alpha_{0}^{2}=\alpha_{p}+\left[\alpha_{p}^{2}+C_{p}^{2}\right]^{1 / 2} .
$$

Таким образом, имеются две стационарные точки: одна при $\alpha>0$ (растяжение) - устойчивый фокус, и другая при $\alpha<0$ (сжатие). Обе точки имеют малые и равные значения $\chi_{0}$. Точка с $\alpha_{0}<0$ является неустойчивым фокусом, а при $\alpha_{0}>0$ точка является устойчивой. Возможно, кому-то покажется странным, что растяжение соответствует притягивающей точке, хотя следует также отметить, что эти уравнения без гессианов возникают при турбулентном моделировании системы Навье-Стокса [87].

Наконец, существование соотношения (6.1), а также его более общего лагранжева эквивалента (3.9) является основным элементом при доказательстве теоремы 1 , из которой выводится динамика реперов. Кроме того, для трехмерных уравнений Эйлера (6.1) служит ключевым шагом при доказательстве теоремы 8.

За плодотворное обсуждение этой работы я хочу поблагодарить Даррил Холм (Darryl Holm), Грега Павлиотиса (Greg Pavliotis), Тревора Стюарта (Trevor Stuart), Аркадия Цинобера (Arkady Tsinober) и Кристоса Вассиликоса (Christos Vassilicos) моих коллег из Королевского Колледжа Лондона, а также Уриела Фриша (Uriel Frisch) из обсерватории Ниццы, Тома Хоy (Tom Hou) из Калифорнийского технологического института, Роберта Keppa (Robert Kerr) 
из Университета Варвика, Яна Рулстона (Ian Roulstone) из Университета Суррея, Эдриса Тити из Института Вейцмана, Израиль, и Владимира Владимирова из Университета Йорка. Я также весьма благодарен за теплый прием организаторам (особенно Андрею Фурсикову и Сергею Куксину) конференции "Математическая гидродинамика", состоявшейся в институте им. В. А. Стеклова в Москве в июне 2006 г.

\section{Список литературы}

[1] J. J. O'Connor, E.F. Robertson, Sir William Rowan Hamilton, http:// www-groups.dcs.st-and.ac.uk/ history/Mathematicians/Hamilton.html, 1998.

[2] A. J. Hanson, Visualizing quaternions, Elsevier, London, 2006.

[3] В.И. Арнольд, Математические методы классической механики, Наука, М., 1974; англ. пер.: V. I. Arnol'd, Mathematical methods of classical mechanics, Grad. Texts in Math., 60, Springer-Verlag, New York-Heidelberg, 1978.

[4] J.E. Marsden, Lectures on geometric methods in mathematical physics, CBMS-NSF Regional Conf. Ser. in Appl. Math., 37, SIAM, Philadelphia, 1981.

[5] J.E. Marsden, Lectures on mechanics, London Math. Soc. Lecture Note Ser., 174, Cambridge Univ. Press, Cambridge, 1992.

[6] A. Cayley, "On certain results being related to quaternions", Phil. Mag., 26 (1845), 141-145.

[7] W. R. Hamilton, Lectures on quaternions, Cambridge Univ. Press, Cambridge, 1853.

[8] W. R. Hamilton, Elements of quaternions, Cambridge Univ. Press, Cambridge, 1866; Chelsea Publ., New York, 1969.

[9] P. G. Tait, An elementary treatise on quaternions, 3rd ed., Cambridge Univ. Press, Cambridge, 1890.

[10] E. T. Whittaker, A treatise on the analytical dynamics of particles and rigid bodies, Dover, New York, 1944.

[11] F. Klein, The mathematical theory of the top. Lectures delivered on the occasion of the sesquicentennial celebration of Princeton University, Dover Phoenix Ed., 2, Dover, Mineola, 2004.

[12] J.B. Kuipers, Quaternions and rotation sequences. A primer with applications to orbits, aerospace, and virtual reality, Princeton Univ. Press, Princeton, 1999.

[13] K. Shoemake, "Animating rotation with quaternion curves", Comput. Graphics, 19:3 (1985), 245-254.

[14] V. I. Arnold, B. A. Khesin, Topological methods in hydrodynamics, Appl. Math. Sci., 125, Springer-Verlag, New York, 1998.

[15] A. J. Majda, A. L. Bertozzi, Vorticity and incompressible flow, Cambridge Texts Appl. Math., 27, Cambridge Univ. Press, Cambridge, 2002.

[16] G. K. Batchelor, An introduction to fluid dynamics, Cambridge Math. Lib., Cambridge Univ. Press, Cambridge, 1999.

[17] P. G. Saffman, Vortex dynamics, Cambridge Monogr. Mech. Appl. Math., Cambridge Univ. Press, Cambridge, 1992.

[18] H. K. Moffatt, S. Kida, K. Ohkitani, "Stretched vortices - the sinews of turbulence; large-Reynolds-number asymptotics", J. Fluid Mech., 259 (1994), 241-264.

[19] A. La Porta, G. A. Voth, A. Crawford, J. Alexander, E. Bodenschatz, "Fluid particle accelerations in fully developed turbulence", Nature, 409 (2001), 1017-1019.

[20] N. Mordant, P. Metz, O. Michel, J.-F. Pinton, "Measurement of Lagrangian velocity in fully developed turbulence", Phys. Rev. Lett., 87:21 (2001), 214501. 
[21] G. A. Voth, A. La Porta, A. Crawford, J. Alexander, E. Bodenschatz, "Measurement of particle accelerations in fully developed turbulence", J. Fluid Mech., 469:1 (2002), 121-160.

[22] N. Mordant, A. M. Crawford, E. Bodenschatz, "Three-dimensional structure of the Lagrangian acceleration in turbulent flows", Phys. Rev. Lett., 93:21 (2004), 214501.

[23] N. Mordant, E. Lévêque, J.-F. Pinton, "Experimental and numerical study of the Lagrangian dynamics of high Reynolds turbulence", New J. Phys., 6:1 (2004), 1-116.

[24] N. Mordant, P. Metz, J.-F. Pinton, O. Michel, "Acoustical technique for Lagrangian velocity measurement", Rev. Sci. Instrum., 76:2 (2005), 025105.

[25] B. A. Lüthi, A. Tsinober, W. Kinzelbach, "Lagrangian measurement of vorticity dynamics in turbulent flow", J. Fluid Mech., 528 (2005), 87-118.

[26] L. Biferale, G. Boffetta, A. Celani, A. Lanotte, F. Toschi, "Particle trapping in threedimensional fully developed turbulence", Phys. Fluids, 17:2 (2005), 021701.

[27] A. M. Reynolds, N. Mordant, A. M. Crawford, E. Bodenschatz, "On the distribution of Lagrangian accelerations in turbulent flows", New J. Phys., 7 (2005), 58.

[28] W. Braun, F. De Lillo, B. Eckhardt, "Geometry of particle paths in turbulent flows", J. Turbul., 7 (2006), 62.

[29] E. Dresselhaus, M. Tabor, "The kinematics of stretching and alignment of material elements in general flow fields", J. Fluid Mech., 236 (1991), 415-444.

[30] H. K. Moffatt, Magnetic field generation in electrically conducting fluids, Cambridge Univ. Press, Cambridge, 1978; pyc. пер.: Г. Моффат, Возбуждение магнитного поля в проводящей среде, Мир, М., 1980.

[31] J. Norbury, I. Roulstone (eds.), Large-scale atmosphere-ocean dynamics, vol. 1, 2, Cambridge Univ. Press, Cambridge, 2002.

[32] A. J. Majda, Introduction to PDEs and waves for the atmosphere and ocean, Courant Lect. Notes Math., 9, Amer. Math. Soc., Providence, 2003.

[33] M. J. P. Cullen, A mathematical theory of large-scale atmosphere/ocean flow, Imperial College Press, London, 2006.

[34] G. Falkovich, K. Gawedzki, M. Vergassola, "Particles and fields in fluid turbulence", Rev. Modern Phys., 73:4 (2001), 913-975.

[35] H. Ertel, "Ein neuer hydrodynamischer Wirbelsatz", Met. Z., 59 (1942), 277-281.

[36] K. Ohkitani, "Eigenvalue problems in three-dimensional Euler flows", Phys. Fluids A, 5:10 (1993), 2570-2572.

[37] В.Е. Захаров, Е.А. Кузнецов, "Гамильтоновский формализм для нелинейных волн", УФН, 167:11 (1997), 1137-1167; англ. пер.: V. E. Zakharov, E. A. Kuznetsov, "Hamiltonian formalism for nonlinear waves", Phys. Usp., 40:11 (1997), 1087-1116.

[38] C. Truesdell, R. A. Toupin, "The classical field theories", Encyclopaedia of physics, vol. 3, part 1, ed. S. Flügge, Springer, Berlin, 1960, 226-793.

[39] A. Viúdez, "On Ertel's potential vorticity theorem. On the impermeability theorem for potential vorticity", J. Atmos. Sci., 56:4 (1999), 507-516.

[40] K. Ohkitani, S. Kishiba, "Nonlocal nature of vortex stretching in an inviscid fluid", Phys. Fluids, 7:2 (1995), 411-421.

[41] В.И. Юдович, "Нестационарные течения идеальной несжимаемой жидкости", ЖВМ и МФ, 3 (1963), 1032-1066.

[42] О.А. Ладыженская, Математические вопросы динамики вязкой несжимаемой жидкости, Физматгиз, М., 1961; англ. пер.: О. A. Ladyzhenskaya, The mathematical theory of viscous incompressible flow, Gordon and Breach, New York, 1963.

[43] P. Constantin, C. Foias, Navier-Stokes equations, Chicago Lectures in Math., Univ. Chicago Press, Chicago, 1988. 
[44] C. Foias, O. Manley, R. Rosa, R. Temam, Navier-Stokes equations and turbulence, Encyclopedia Math. Appl., 83, Cambridge Univ. Press, Cambridge, 2001.

[45] J. T. Stuart, "Nonlinear Euler partial differential equations: singularities in their solution", Applied mathematics, fluid mechanics, astrophysics (Cambridge, MA, USA, 1987), eds. D. J. Benney, F. H. Shu, Chi Yuan, World Sci. Publ., Singapore, 1988, 81-95.

[46] J. T. Stuart, "The Lagrangian picture of fluid motion and its implication for flow structures", IMA J. Appl. Math., 46:1-2 (1991), 147-163.

[47] J. T. Stuart, "Singularities in three-dimensional compressible Euler flows with vorticity", Theoret. Comput. Fluid Dyn., 10:1-4 (1998), 385-391.

[48] J.D. Gibbon, A. Fokas, C.R. Doering, "Dynamically stretched vortices as solutions of the 3D Navier-Stokes equations", Phys. D, 132:4 (1999), 497-510.

[49] K. Ohkitani, J.D. Gibbon, "Numerical study of singularity formation in a class of Euler and Navier-Stokes flows", Phys. Fluids, 12:12 (2000), 3181-3194.

[50] P. Constantin, "The Euler equations and nonlocal conservative Riccati equations", Internat. Math. Res. Notices, 2000, № 9, 455-465.

[51] J. D. Gibbon, D. R. Moore, J. T. Stuart, "Exact, infinite energy, blow-up solutions of the three-dimensional Euler equations", Nonlinearity, 16:5 (2003), 1823-1831.

[52] J. T. Beale, T. Kato, A. Majda, "Remarks on the breakdown of smooth solutions for the 3D Euler equations", Comm. Math. Phys., 94:1 (1984), 61-66.

[53] G. Ponce, "Remarks on a paper ['Remarks on the breakdown of smooth solutions for the 3D Euler equations'] by J. T. Beale, T. Kato and A. Majda", Comm. Math. Phys., 98:3 (1985), 349-353.

[54] H. Kozono, Y. Taniuchi, "Limiting case of the Sobolev inequality in BMO, with applications to the Euler equations", Comm. Math. Phys., 214:1 (2000), 191-200.

[55] P. Constantin, "Geometric statistics in turbulence", SIAM Rev., 36:1 (1994), 73-98.

[56] P. Constantin, C. Fefferman, A. Majda, "Geometric constraints on potentially singular solutions for the 3-D Euler equation", Comm. Partial Differential Equations, 21:3-4 (1996), 559-571.

[57] J. Deng, T. Y. Hou, X. Yu, "Geometric properties and non-blowup of 3D incompressible Euler flow", Comm. Partial Differential Equations, 30:1-2 (2005), 225-243.

[58] J. Deng, T. Y. Hou, X. Yu, "Improved geometric condition for non-blowup of the 3D incompressible Euler equation", Comm. Partial Differential Equations, 31:2 (2006), 293-306.

[59] J. D. Gibbon, D. D. Holm, R. M. Kerr, I. Roulstone, "Quaternions and particle dynamics in Euler fluid flow", Nonlinearity, 19:8 (2006), 1969-1983.

[60] A. Sudbery, "Quaternionic analyis", Math. Proc. Cambridge Philos. Soc., 85:2 (1979), 199-225.

[61] A. S. Fokas, D. A. Pinotsis, "Quaternions, evaluation of integrals and boundary value problems", Comput. Methods Funct. Theory (to appear).

[62] J.D. Gibbon, D. D. Holm, Lagrangian particle paths and ortho-normal quaternion frames, arXiv: nlin. CD/0607020.

[63] B. Galanti, J. D. Gibbon, M. Heritage, "Vorticity alignment results for the threedimensional Euler and Navier-Stokes equations", Nonlinearity, 10:6 (1997), 16751694.

[64] J. D. Gibbon, B. Galanti, R. M. Kerr, R. M., "Stretching and compression of vorticity in the 3D Euler equations", Turbulence structure and vortex dynamics (Cambridge, 1999), eds. J. C. R. Hunt, J. C. Vassilicos, Cambridge Univ. Press, Cambridge, 2000, 23-34.

[65] J.D. Gibbon, "A quaternionic structure in the three-dimensional Euler and ideal magneto-hydrodynamics equation", Phys. D, 166:1-2 (2002), 17-28. 
[66] D. Chae, "Remarks on the blow-up of the Euler equations and the related equations", Comm. Math. Phys., 245:3 (2004), 539-550.

[67] D. Chae, "On the finite time singularities of the 3D incompressible Euler equations", Comm. Pure Appl. Math., 60:4 (2007), 597-617.

[68] M. E. Brachet, D. I. Meiron, S. A. Orszag, B. G. Nickel, R. H. Morf, U. Frisch, "Smallscale structure of the Taylor-Green vortex", J. Fluid Mech., 130 (1983), 411-452.

[69] M. E. Brachet, V. Meneguzzi, A. Vincent, H. Politano, P.-L. Sulem, "Numerical evidence of smooth self-similar dynamics and possibility of subsequent collapse for threedimensional ideal flows", Phys. Fluids A, 4:12 (1992), 2845-2854.

[70] A. Pumir, E. Siggia, "Collapsing solutions to the 3-D Euler equations", Phys. Fluids A, 2:2 (1990), 220-241.

[71] R. M. Kerr, "Evidence for a singularity of the three-dimensional, incompressible Euler equations", Phys. Fluids A, 5:7 (1993), 1725-1746.

[72] R. M. Kerr, "Vorticity and scaling of collapsing Euler vortices", Phys. Fluids, 17:7 (2005), 075103.

[73] R. Grauer, C. Marliani, K. Germaschewski, "Adaptive mesh refinement for singular solutions of the incompressible Euler equations", Phys. Rev. Lett., 80:19 (1998), 41774180.

[74] O. N. Boratav, R. B. Pelz, "Direct numerical simulation of transition to turbulence from a high-symmetry initial condition", Phys. Fluids, 6:8 (1994), 2757-2784.

[75] R. B. Pelz, "Symmetry and the hydrodynamic blow-up problem", J. Fluid Mech., 444 (2001), 299-320.

[76] T. Y. Hou, R. Li, "Dynamic depletion of vortex stretching and non-blowup of the 3-D incompressible Euler equations", J. Nonlinear Sci., 16:6 (2006), 639-664.

[77] W. Pauls, T. Matsumoto, U. Frisch, J. Bec, "Nature of complex singularities for the 2D Euler equation", Phys. D, 219:1 (2006), 40-59.

[78] T. Kato, "Nonstationary flows of viscous and ideal fluids in $\mathbb{R}^{3}$ ", J. Funct. Anal., 9:3 (1972), 296-305.

[79] R. M. Kerr, Computational Euler history, arXiv: physics/0607148.

[80] D. Cordoba, C. Fefferman, "On the collapse of tubes carried by 3D incompressible flows", Comm. Math. Phys., 222:2 (2001), 293-298.

[81] A. Shnirelman, "On the nonuniqueness of weak solution of the Euler equation", Comm. Pure Appl. Math., 50:12 (1997), 1261-1286.

[82] E. Tadmor, "On a new scale of regularity spaces with applications to Euler's equations", Nonlinearity, 14:3 (2001), 513-532.

[83] D. Chae, "On the Euler equations in the critical Triebel-Lizorkin spaces", Arch. Ration. Mech. Anal., 170:3 (2003), 185-210.

[84] D. Chae, "Local existence and blow-up criterion for the Euler equations in the Besov spaces", Asymptot. Anal., 38:3-4 (2004), 339-358.

[85] D. Chae, "Remarks on the blow-up criterion of the three-dimensional Euler equations", Nonlinearity, 18:3 (2005), 1021-1029.

[86] J.D. Gibbon, D. D. Holm, Lagrangian analysis of alignment dynamics for isentropic compressible magnetohydrodynamics, arXiv: nlin.CD/0608009.

[87] Li Yi, C. Meneveau, "Origin of non-Gaussian statistics in hydrodynamic turbulence", Phys. Rev. Lett., 95:16 (2005), 164502.

Дж. Д. Гиббон (J. D. Gibbon)

Imperial College London, UK

Поступила в редакцию

E-mail: j.d.gibbon@ic.ac.uk

27.09 .2006 